\title{
Central limit theorem for locally interacting Fermi gas
}

\author{
V. Jakšić ${ }^{1}$, Y. Pautrat ${ }^{2}$, C.-A. Pillet ${ }^{3}$ \\ ${ }^{1}$ Department of Mathematics and Statistics \\ McGill University \\ 805 Sherbrooke Street West \\ Montreal, QC, H3A 2K6, Canada \\ ${ }^{2}$ Univ. Paris-Sud \\ Laboratoire de Mathématiques d'Orsay \\ Orsay cedex, F-91405, France \\ ${ }^{3}$ Centre de Physique Théorique* \\ Université du Sud Toulon-Var, B.P. 20132 \\ 83957 La Garde Cedex, France
}

June 18, 2008

\begin{abstract}
We consider a locally interacting Fermi gas in its natural non-equilibrium steady state and prove the Quantum Central Limit Theorem (QCLT) for a large class of observables. A special case of our results concerns finitely many free Fermi gas reservoirs coupled by local interactions. The QCLT for flux observables, together with the Green-Kubo formulas and the Onsager reciprocity relations previously established [JOP4], complete the proof of the Fluctuation-Dissipation Theorem and the development of linear response theory for this class of models.
\end{abstract}

*UMR 6207, CNRS, Université de la Méditerranée, Université de Toulon et Université de Provence 


\section{Introduction}

This paper and its companion [AJPP3] are first in a series of papers dealing with fluctuation theory of non-equilibrium steady states in quantum statistical mechanics. They are part of a wider program initiated in [Ru2, Ru3, JP1, JP2, JP4] which deals with the development of a mathematical theory of non-equilibrium statistical mechanics in the framework of algebraic quantum statistical mechanics [BR1, BR2, Pi]. For additional information about this program we refer the reader to the reviews [Ru4, JP3, AJPP1].

In this paper we study the same model as in [JOP4]: A free Fermi gas in a quasi-free state perturbed by a sufficiently regular local interaction. It is well-known that under the influence of such a perturbation this system approaches, as time $t \rightarrow+\infty$, a steady state commonly called the natural non-equilibrium steady state (NESS) [BM1, AM, BM2, FMU, JOP4]. Our main result is that under very general conditions the Quantum Central Limit Theorem (QCLT) holds for this NESS. Combined with the results of [JOP4], the QCLT completes the proof of the near-equilibrium Fluctuation-Dissipation Theorem and the development of linear response theory for this class of models.

The rest of this introduction is organized as follows. In Subsection 1.1 for notational purposes we review a few basic concepts of algebraic quantum statistical mechanics. In this subsection the reader can find the definition of QCLT for quantum dynamical systems and a brief review of related literature. Our main result is stated in Subsection 1.2. In Subsection 1.3 we discuss our results in the context of linear response theory.

Acknowledgment. A part of this work has been done during Y.P.'s stay at McGill University and the C.R.M. as ISM Postdoctoral Fellow, during his visit to McGill University funded by NSERC and his visit to Erwin Schrödinger Institut. The research of V.J. was partly supported by NSERC. We wish to thank Manfred Salmhofer for useful discussions.

\subsection{Central limit theorem for quantum dynamical systems}

Let $\mathcal{O}$ be a $C^{*}$-algebra with identity $\mathbb{1}$ and let $\tau^{t}, t \in \mathbb{R}$, be a strongly continuous group of $*$-automorphisms of $\mathcal{O}$. The pair $(\mathcal{O}, \tau)$ is called a $C^{*}$-dynamical system. A positive normalized element of the dual $\mathcal{O}^{*}$ is called a state on $\mathcal{O}$. In what follows $\omega$ is a given $\tau$-invariant state on $\mathcal{O}$. The triple $(\mathcal{O}, \tau, \omega)$ is called a quantum dynamical system.

The system $(\mathcal{O}, \tau, \omega)$ is called ergodic if

$$
\lim _{t \rightarrow \infty} \frac{1}{t} \int_{0}^{t} \omega\left(B^{*} \tau^{s}(A) B\right) \mathrm{d} s=\omega\left(B^{*} B\right) \omega(A),
$$

and mixing if

$$
\lim _{|t| \rightarrow \infty} \omega\left(B^{*} \tau^{t}(A) B\right)=\omega\left(B^{*} B\right) \omega(A)
$$

for all $A, B \in \mathcal{O}$.

We denote by $\left(\mathcal{H}_{\omega}, \pi_{\omega}, \Omega_{\omega}\right)$ the GNS-representation of the $C^{*}$-algebra $\mathcal{O}$ associated to the state $\omega$. The state $\omega$ is called modular if $\Omega_{\omega}$ is a separating vector for the enveloping von Neumann algebra $\pi_{\omega}(\mathcal{O})^{\prime \prime}$. The states of thermal equilibrium are described by the $(\tau, \beta)$-KMS condition where $\beta>0$ is the inverse temperature. Any $(\tau, \beta)$-KMS state on $\mathcal{O}$ is $\tau$-invariant and modular.

For any subset $\mathcal{A} \subset \mathcal{O}$ we denote by $\mathcal{A}_{\text {self }}=\left\{A \in \mathcal{A} \mid A=A^{*}\right\}$ the set of self-adjoint elements of $\mathcal{A}$. Let $f$ be a bounded Borel function on $\mathbb{R}$ and $A \in \mathcal{O}_{\text {self }}$. With a slight abuse of notation in the sequel we will often denote $f\left(\pi_{\omega}(A)\right)$ by $f(A)$ and write $\omega(f(A))=\left(\Omega_{\omega}, f\left(\pi_{\omega}(A)\right) \Omega_{\omega}\right)$. With this convention, $1_{[a, b]}(A)$ denotes the spectral projection on the interval $[a, b]$ of $\pi_{\omega}(A)$. We shall use the same convention for the products $f_{1}\left(\pi_{\omega}\left(A_{1}\right)\right) \cdots f_{n}\left(\pi_{\omega}\left(A_{n}\right)\right)$, etc.

An involutive antilinear $*$-automorphism $\Theta$ of $\mathcal{O}$ is called time-reversal if $\Theta \circ \tau^{t}=\tau^{-t} \circ \Theta$. A state $\eta$ on $\mathcal{O}$ is called time-reversal invariant if $\eta \circ \Theta(A)=\eta\left(A^{*}\right)$ holds for all $A \in \mathcal{O}$.

We say that a subset $\mathcal{A} \subset \mathcal{O}$ is $L^{1}$-asymptotically abelian for $\tau$ if for all $A, B \in \mathcal{A}$,

$$
\int_{-\infty}^{\infty}\left\|\left[A, \tau^{t}(B)\right]\right\| \mathrm{d} t<\infty .
$$


Throughout the paper we shall use the shorthand

$$
\tilde{A}_{t} \equiv \frac{1}{\sqrt{t}} \int_{0}^{t}\left(\tau^{s}(A)-\omega(A)\right) \mathrm{d} s .
$$

Definition 1.1 Let $\mathfrak{C}$ be a $*$-vector subspace of $\mathcal{O}$. We say that $\mathfrak{C}$ is CLT-admissible iffor all $A, B \in \mathfrak{C}$,

$$
\int_{-\infty}^{\infty}\left|\omega\left(\tau^{t}(A) B\right)-\omega(A) \omega(B)\right| \mathrm{d} t<\infty .
$$

For $A, B \in \mathfrak{C}$ we set

$$
\begin{gathered}
L(A, B) \equiv \int_{-\infty}^{\infty} \omega\left(\left(\tau^{t}(A)-\omega(A)\right)(B-\omega(B))\right) \mathrm{d} t=\int_{-\infty}^{\infty}\left(\omega\left(\tau^{t}(A) B\right)-\omega(A) \omega(B)\right) \mathrm{d} t \\
\varsigma(A, B) \equiv \frac{1}{2 \mathrm{i}} \int_{-\infty}^{\infty} \omega\left(\left[\tau^{t}(A), B\right]\right) \mathrm{d} t=\frac{1}{2 \mathrm{i}}(L(A, B)-L(B, A)) .
\end{gathered}
$$

The functional $(A, B) \mapsto L(A, B)$ is obviously bilinear. Other properties of this functional are summarized in:

Proposition 1.2 Suppose that $\mathfrak{C}$ is CLT-admissible and let $A, B \in \mathfrak{C}$. Then:

(i) $L\left(A^{*}, A\right) \geq 0$.

(ii) $L(A, B)=\overline{L\left(B^{*}, A^{*}\right)}$. In particular, if $A$ and $B$ are self-adjoint, then $\varsigma(A, B)=\operatorname{Im} L(A, B)$.

(iii) $\left|L\left(A^{*}, B\right)\right|^{2} \leq L\left(A^{*}, A\right) L\left(B^{*}, B\right)$.

(iv) $(A, B) \mapsto \varsigma(A, B)$ is a (possibly degenerate) symplectic form on the real vector space $\mathfrak{C}_{\text {self. }}$.

(v) If $\omega$ is a mixing $(\tau, \beta)$-KMS state, then $\varsigma=0$.

(vi) Suppose that $\varsigma=0$, that $\mathfrak{C}$ is dense in $\mathcal{O}$ and $L^{1}$-asymptotically abelian for $\tau$, and that $\omega$ is either a factor state or 3-fold mixing: For all $A_{1}, A_{2}, A_{3} \in \mathcal{O}$,

$$
\lim _{\min _{i \neq j}\left|t_{i}-t_{j}\right| \rightarrow \infty} \omega\left(\tau^{t_{1}}\left(A_{1}\right) \tau^{t_{2}}\left(A_{2}\right) \tau^{t_{3}}\left(A_{3}\right)\right)=\omega\left(A_{1}\right) \omega\left(A_{2}\right) \omega\left(A_{3}\right) .
$$

Then $\omega$ is a $(\tau, \beta)$-KMS state for some $\beta \in \mathbb{R} \cup\{ \pm \infty\}$.

Proof. Note that

$$
0 \leq \omega\left(\tilde{A}_{t}^{*} \tilde{A}_{t}\right)=\int_{-t}^{t}\left(1-\frac{|s|}{t}\right) \omega\left(\left(\tau^{t}\left(A^{*}\right)-\omega\left(A^{*}\right)\right)(A-\omega(A))\right) \mathrm{d} s .
$$

This identity and the dominated convergence theorem yield

$$
L\left(A^{*}, A\right)=\lim _{t \rightarrow \infty} \omega\left(\tilde{A}_{t}^{*} \tilde{A}_{t}\right) \geq 0,
$$

and (i) follows. Parts (ii) and (iv) are obvious. (i) and (ii) imply the Cauchy-Schwartz inequality (iii). Part (v) follows from Proposition 5.4.12 in [BR2]. Part (vi) is the celebrated stability result of Bratteli, Kishimoto and Robinson [BKR], see Proposition 5.4.20 in [BR2].

Definition 1.3 Let $\mathfrak{C}$ be CLT-admissible. We shall say that the Simple Quantum Central Limit Theorem (SQCLT) holds for $\mathfrak{C}$ w.r.t. $(\mathcal{O}, \tau, \omega)$ if for all $A \in \mathfrak{C}_{\text {self, }}$

$$
\lim _{t \rightarrow \infty} \omega\left(\mathrm{e}^{\mathrm{i} \tilde{A}_{t}}\right)=\exp \left(-\frac{1}{2} L(A, A)\right)
$$

We shall say that the Quantum Central Limit Theorem $(Q C L T)$ holds for $\mathfrak{C}$ if for all $n$ and all $A_{1}, \cdots, A_{n}$ in $\mathfrak{C}_{\text {self }}$,

$$
\lim _{t \rightarrow \infty} \omega\left(\mathrm{e}^{\mathrm{i} \tilde{A}_{1 t}} \cdots \mathrm{e}^{\mathrm{i} \tilde{A}_{n t}}\right)=\exp \left(-\frac{1}{2} \sum_{1 \leq j, k \leq n} L\left(A_{k}, A_{j}\right)-\mathrm{i} \sum_{1 \leq j<k \leq n} \varsigma\left(A_{j}, A_{k}\right)\right) .
$$


The SQCLT is obviously a special case of the QCLT. Under sufficient ergodic assumptions, however, the QCLT can be deduced from the SQCLT.

Theorem 1.4 Suppose that $\mathfrak{C}$ is CLT-admissible and $L^{1}$-asymptotically abelian for $\tau$. Suppose also that the system $(\mathcal{O}, \tau, \omega)$ is ergodic and that the state $\omega$ is modular. If the SQCLT holds for $\mathfrak{C}$ w.r.t. $(\mathcal{O}, \tau, \omega)$ then the QCLT also holds for $\mathfrak{C}$.

We shall prove Theorem 1.4 in Section 2 following the ideas of [GV].

The SQCLT has the same probabilistic interpretation as the classical central limit theorem. The probability of measuring a value of $A$ in $[a, b]$ when the system is in the state $\omega$ is given by

$$
\operatorname{Prob}_{\omega}\{A \in[a, b]\}=\omega\left(1_{[a, b]}(A)\right) .
$$

If SQCLT holds for $A$, then

$$
\lim _{t \rightarrow \infty} \operatorname{Prob}_{\omega}\left\{\frac{1}{t} \int_{0}^{t} \tau^{s}(A) \mathrm{d} s \in \omega(A)+\left[\frac{a}{\sqrt{t}}, \frac{b}{\sqrt{t}}\right]\right\}=\frac{1}{\sqrt{2 \pi L(A, A)}} \int_{a}^{b} \mathrm{e}^{-x^{2} / 2 L(A, A)^{2}} \mathrm{~d} x .
$$

Except in trivial cases, the QCLT does not have a classical probabilistic interpretation. In this case the relevant concept is the $\mathrm{CCR}$ algebra over the symplectic space $\left(\mathfrak{C}_{\text {self }}, \varsigma\right)$, often called the fluctuation algebra [GVV1]. The mathematical structure of the fluctuation algebra is discussed in many places in the literature, see e.g. [GVV1]-[GVV6] and [MSTV, BR2, Pe, OP, De2] for general results about CCR algebras. For notational and reference purposes we recall a few basic facts. Let $\mathcal{W}$ be the $C^{*}$-algebra generated by the elements $\left\{W(A) \mid A \in \mathfrak{C}_{\text {self }}\right\}$ such that for all $A, B$ in $\mathfrak{C}_{\text {self }}$

$$
W(-A)=W(A)^{*}, \quad W(A) W(B)=\mathrm{e}^{-\mathrm{i} \varsigma(A, B) / 2} W(A+B),
$$

equipped with the minimal regular norm. The map

$$
\omega_{L}(W(A)) \equiv \mathrm{e}^{-L(A, A) / 2},
$$

uniquely extends to a quasi-free state on $\mathcal{W}$ and (1.1) can be written as

$$
\lim _{t \rightarrow \infty} \omega\left(\mathrm{e}^{\mathrm{i} \tilde{A}_{1 t}} \cdots \mathrm{e}^{\mathrm{i} \tilde{A}_{n t}}\right)=\omega_{L}\left(W\left(A_{1}\right) \cdots W\left(A_{n}\right)\right) .
$$

The pair $\left(\mathcal{W}, \omega_{L}\right)$ describes the fluctuations of $\mathfrak{C}$ w.r.t. the quantum dynamical system $(\mathcal{O}, \tau, \omega)$. Let $\left(\mathcal{H}_{L}, \pi_{L}, \Omega_{L}\right)$ be the GNS representation of $\mathcal{W}$ associated to $\omega_{L}$. We shall also denote by $\omega_{L}$ the induced state on the enveloping von Neumann algebra $\pi_{L}(\mathcal{W})^{\prime \prime}$. Since for all $A \in \mathfrak{C}_{\text {self }}$ the map

$$
\mathbb{R} \ni x \mapsto \omega_{L}(W(x A)),
$$

extends to an entire analytic function on $\mathbb{C}$, there exist self-adjoint operators $\varphi_{L}(A)$ on $\mathcal{H}_{L}$ such that

$$
\pi_{L}(W(A))=\mathrm{e}^{\mathrm{i} \varphi_{L}(A)} .
$$

Moreover, the operators $\varphi_{L}(A), A \in \mathfrak{C}_{\text {self }}$ have a common dense set of analytic vectors $\mathcal{A} \subset \mathcal{H}_{L}$ and on this set

$$
\left[\varphi_{L}(A), \varphi_{L}(B)\right]=\mathrm{i} \varsigma(A, B) \mathbb{1} .
$$

The operators $\varphi_{L}(A)$ are the Bose fields associated by QCLT to $(\mathcal{O}, \tau, \omega)$. For any $n$ and $A_{1}, \cdots, A_{n} \in \mathfrak{C}_{\text {self }}, \Omega_{L}$ is in the domain of $\varphi_{L}\left(A_{1}\right) \cdots \varphi_{L}\left(A_{n}\right)$ and, as usual, we denote

$$
\omega_{L}\left(\varphi_{L}\left(A_{1}\right) \cdots \varphi_{L}\left(A_{n}\right)\right) \equiv\left(\Omega_{L}, \varphi_{L}\left(A_{1}\right) \cdots \varphi_{L}\left(A_{n}\right) \Omega_{L}\right) .
$$

In particular $\omega_{L}\left(\varphi_{L}\left(A_{1}\right) \varphi_{L}\left(A_{2}\right)\right)=L\left(A_{1}, A_{2}\right)$. For any integer $n$ we denote $\mathcal{P}_{n}$ the set of all permutations $\pi$ of $\{1, \ldots, 2 n\}$ such that

$$
\pi(2 j-1)<\pi(2 j), \quad \text { and } \quad \pi(2 j-1)<\pi(2 j+1),
$$

for every $j \in\{1, \ldots, n\}$. The cardinality of $\mathcal{P}_{n}$ is $(2 n) ! /\left(2^{n} n !\right)$. Then

$$
\omega_{L}\left(\varphi_{L}\left(A_{1}\right) \cdots \varphi_{L}\left(A_{n}\right)\right)=\left\{\begin{array}{cl}
\sum_{\pi \in \mathcal{P}_{n / 2}} \prod_{j=1}^{n / 2} \omega_{L}\left(\varphi_{L}\left(A_{\pi(2 j-1)}\right) \varphi_{L}\left(A_{\pi(2 j)}\right)\right), & \text { if } n \text { is even; } \\
0, & \text { if } n \text { is odd. }
\end{array}\right.
$$

The QCLT and the non-commutative Lévy-Cramér theorem proven in [JPP] yield: 
Theorem 1.5 Suppose that $Q C L T$ holds for $\mathfrak{C}$ w.r.t. $(\mathcal{O}, \tau, \omega)$, let $A_{1}, \cdots, A_{n} \in \mathfrak{C}_{\text {self }}$, and let $I_{1}, \ldots, I_{n} \subset \mathbb{R}$ be open intervals. If $L\left(A_{j}, A_{j}\right)=0$, we assume in addition that 0 is not an endpoint of $I_{j}$. Then

$$
\lim _{t \rightarrow \infty} \omega\left(\chi_{I_{1}}\left(\tilde{A}_{1 t}\right) \cdots \chi_{I_{n}}\left(\tilde{A}_{n t}\right)\right)=\omega_{L}\left(\chi_{I_{1}}\left(\varphi_{L}\left(A_{1}\right)\right) \cdots \chi_{I_{n}}\left(\varphi_{L}\left(A_{n}\right)\right)\right)
$$

where $\chi_{I}$ denotes the characteristic function of the interval $I$.

For a probabilistic interpretation of Theorem 1.5 in the context of repeated quantum-mechanical measurements we refer the reader to Section 2 in [Da1].

The QCLT does not imply that

$$
\lim _{t \rightarrow \infty} \omega\left(\tilde{A}_{1 t} \cdots \tilde{A}_{n t}\right)=\omega_{L}\left(\varphi_{L}\left(A_{1}\right) \cdots \varphi_{L}\left(A_{n}\right)\right),
$$

and in principle the convergence of moments has to be established separately. In our model, the proof of (1.8) is an intermediate step in the proof of the QCLT.

To define Bose annihilation and creation operators associated with fields $\varphi_{L}(A)$, we need to assume that the symplectic form $\varsigma$ is non-degenerate and that $\mathfrak{C}_{\text {self }}$ is either even- or infinite-dimensional. In this case there exists a complex structure $J$ on $\mathfrak{C}_{\text {self }}$ satisfying $\varsigma(J A, J B)=\varsigma(A, B)$, and one can define the operators $a_{L}(A) / a_{L}^{*}(A)$ on $\mathcal{A}$ by

$$
a_{L}(A) \equiv \frac{1}{\sqrt{2}}\left(\varphi_{L}(A)+\mathrm{i} \varphi_{L}(J A)\right), \quad a_{L}^{*}(A) \equiv \frac{1}{\sqrt{2}}\left(\varphi_{L}(A)-\mathrm{i} \varphi_{L}(J A)\right) .
$$

These operators are closable and satisfy

$$
\left[a_{L}(A), a_{L}^{*}(B)\right]=\mathrm{i}(\varsigma(A, B)-\mathrm{i} \varsigma(A, J B)),
$$

on $\mathcal{A}$.

We expect that in typical physical examples the symplectic form $\varsigma$ will be degenerate in which case the Bose annihilation and creation operators (1.9) cannot be defined globally. Let us consider first the extreme case $\varsigma=0$ (this will hold, for example, if $\omega$ is a mixing $(\tau, \beta)$-KMS state). Let $\hat{\mathfrak{C}}_{\text {self }}$ be the group of all characters of the discrete Abelian group $\mathfrak{C}_{\text {self }}$. The dual group $\hat{\mathfrak{C}}_{\text {self }}$ endowed with the topology of pointwise convergence is a compact topological group and the algebra $\mathcal{W}$ is isomorphic to the $C^{*}$-algebra of all continuous functions on $\hat{\mathfrak{C}}_{\text {self }}$. The state $\omega_{L}$ is identified with the Gaussian measure on $\hat{\mathfrak{C}}_{\text {self }}$ uniquely determined by

$$
\int \chi(A) \mathrm{d} \mu_{L}(\chi)=\mathrm{e}^{-L(A, A) / 2}
$$

More generally, let

$$
\mathfrak{C}_{\text {self }}^{(1)}=\left\{A \mid \varsigma(A, B)=0 \text { for all } B \in \mathfrak{C}_{\text {self }}\right\},
$$

and suppose that there exist $\mathfrak{C}_{\text {self }}^{(2)}$ such that $\mathfrak{C}_{\text {self }}=\mathfrak{C}_{\text {self }}^{(1)} \oplus \mathfrak{C}_{\text {self }}^{(2)}$ as an orthogonal sum (this is certainly the case if $\mathfrak{C}_{\text {self }}$ is finite dimensional, i.e., we consider QCLT with respect to finitely many observables). The restriction of $\varsigma$ to $\mathfrak{C}_{\mathrm{self}}^{(2)}$ is non-degenerate, and if $W^{(j)}, \omega_{L}^{(j)}, j=1,2$ denote the respective CCR algebras and quasi-free states, then

$$
\mathcal{W}=\mathcal{W}^{(1)} \otimes \mathcal{W}^{(2)}, \quad \omega_{L}=\omega_{L}^{(1)} \otimes \omega_{L}^{(2)} .
$$

In particular, annihilation and creation operators can be associated to the elements of $\mathcal{W}^{(2)}$.

Besides QCLT one may consider the related and more general existence problem for the quantum hydrodynamic limit (QHL). For $\epsilon>0$ and $t>0$, let

$$
\hat{A}_{\epsilon}(t) \equiv \epsilon \int_{0}^{t / \epsilon^{2}}\left(\tau^{s}(A)-\omega(A)\right) \mathrm{d} s .
$$

We say that $\mathfrak{C}$ has QHL w.r.t. $(\mathcal{O}, \tau, \omega)$ if for all $A_{1}, \cdots A_{n} \in \mathfrak{C}_{\text {self }}$, and all $t_{1}>0, \cdots, t_{n}>0$,

$$
\lim _{\epsilon \downarrow 0} \omega\left(\mathrm{e}^{\mathrm{i} \hat{A}_{1 \epsilon}\left(t_{1}\right)} \cdots \mathrm{e}^{\mathrm{i} \hat{A}_{n \epsilon}\left(t_{n}\right)}\right)=\omega_{L}\left(W\left(\chi_{\left[0, t_{1}\right]} \otimes A_{1}\right) \cdots W\left(\chi_{\left[0, t_{n}\right]} \otimes A_{n}\right)\right),
$$

where, in the definition of the Weyl algebra, the bilinear form $L$ must be replaced by

$$
L_{\mathrm{QHL}}\left(\chi_{[0, s]} \otimes A, \chi_{[0, t]} \otimes B\right)=\inf (s, t) L(A, B) .
$$


The special case where all $t_{j}$ 's are equal corresponds to QCLT. The QHL is interpreted as the weak convergence of the quantum stochastic process $\hat{A}_{\epsilon}(t)$ to a quantum Brownian motion. With the obvious reformulation, Theorem 1.5 holds for QHL. Convergence of moments

$$
\lim _{\epsilon \downarrow 0} \omega\left(\hat{A}_{1 \epsilon}\left(t_{1}\right) \cdots \hat{A}_{n \epsilon}\left(t_{n}\right)\right)=\omega_{L}\left(\varphi_{L}\left(\chi_{\left[0, t_{1}\right]} \otimes A_{1}\right) \cdots \varphi_{L}\left(\chi_{\left[0, t_{n}\right]} \otimes A_{n}\right),\right.
$$

is of independent interest. Even more generally, one may associate to a class $\mathfrak{F}$ of real valued integrable functions on $\mathbb{R}$ the observables

with $f \in \mathfrak{F}, A \in \mathfrak{C}$ and study the limit $\epsilon \downarrow 0$ of

$$
\hat{A}_{\epsilon}(f) \equiv \epsilon^{-1} \int_{0}^{\infty} f\left(\epsilon^{2} t\right)\left(\tau^{t}(A)-\omega(A)\right) \mathrm{d} t
$$

$$
\omega\left(\mathrm{e}^{\mathrm{i} \hat{A}_{1 \epsilon}\left(f_{1}\right)} \cdots \mathrm{e}^{\mathrm{i} \hat{A}_{n \epsilon}\left(f_{n}\right)}\right) .
$$

Note that QHL corresponds to the choice $\mathfrak{F}=\left\{\chi_{[0, t]} \mid t>0\right\}$. For reasons of space and notational simplicity we will focus in the paper on the QCLT for locally interacting fermionic systems. With only notational changes our proofs can be extended to establish QHL and (1.11). It is likely that the proofs can be extended to a much larger class of functions $\mathfrak{F}$, but we shall not pursue this question here (see [De1] for a related discussion).

We finish this subsection with a few general remarks. We finish this section with a few remarks about earlier quantum centrallimit type results.

First, notice that, since the law of one single observable is well-defined, the description of the limiting law of a family $\left(\tilde{A}_{x}\right)_{x \geq 0}$ of observables as the parameter $x \rightarrow \infty$, is covered by the classical Lévy- Cramèr theorem. Several results of interest exist, which are only of quantum nature insofar as the computation of the $\operatorname{limit}_{\lim } \rightarrow \infty \omega\left(e^{\mathrm{i} \alpha \tilde{A}_{x}}\right)$ is made more complicated by the quantum setting.

Truly quantum central limit theorems therefore involve an attempt to describe the limiting joint behavior of the law of a family $\left(\tilde{A}^{(1)}, \ldots, \tilde{A}^{(p)}\right)_{x}$ of observables as $x \rightarrow \infty$. The earliest results of this type were obtained in a quantum probabilistic approach and were non commutative analogues of classical results concerning sums of independent, identically distributed variables. Such results can be translated in a physical setting as applying to space fluctuations of one-site observables in quantum spin systems with respect to translation-invariant product states. The generality of the framework and the formulation of the limit vary. We mention in particular [Me] where matrix elements of approximate Weyl operators constructed from Pauli matrices are considered; [GvW] which holds in the general *-algebra case but where only convergence of moments is proved; [Kup] which works in a general $\mathrm{C}^{*}$-algebra setting and where a true convergence in distribution (to a classical Gaussian family) is proved, but only with respect to a tracial state. We also mention $[\mathrm{CH}]$ which, although not a central limit theorem, is a first attempt to characterize a convergence in distribution of a family of non-commutating operators, in terms of a (pseudo)-characteristic function.

The papers [GVV1]-[GVV6] aim at more physical applications: a satisfactory algebra of fluctuations is constructed for space fluctuations of local observables in a quantum spin system with a tranlation-invariant state. That state does not have to be a product state; however, the ergodic assumptions on that state are so strong that no nontrivial application was found beyond the product case. However, these papers were a conceptual improvement and our construction owes much to them. The papers [Ma1]-[Ma2] had a similar spirit but, using less stringent ergodic conditions, gave non trivial application to space fluctuations of local observables in XY chains.

A distinct feature of our work is that we study QCLT with respect to the group $\tau^{t}$ describing the microscopic dynamics of the system. There is a number of technical and conceptual aspects of QCLT which are specific to the dynamical group. For example, the ergodic properties of the system (laws of large numbers), which have to be established prior to study its fluctuations, are typically much harder to prove for the dynamical group than for the lattice translation group. As for the conceptual differences, we mention that if $\omega$ is a $(\tau, \beta)$-KMS state, then by Proposition $1.2(\mathrm{v}), \varsigma=0$ and the CCR algebra of fluctuations $\mathcal{W}$ is commutative (Part (vi) provides a partial converse to this statement). This is in sharp contrast with QCLT w.r.t. the translation group, where even in the simple example of product states of spin systems the fluctuation algebra is non-commutative.

The CLT for classical dynamical systems is discussed in [Li]. For a review of results on dynamical CLT for interacting particle systems in classical statistical mechanics we refer the reader to [Sp] and [KL]. The CLT for classical spin systems is discussed in Section V.7 of [E].

After this paper was completed, we have learned of the work [De1] which is technically and conceptually related to ours. We shall comment on Dereziński's result at the end of Subsection 3.3. 


\subsection{QCLT for locally interacting fermions}

A free Fermi gas is described by the $C^{*}$-dynamical system $\left(\mathcal{O}, \tau_{0}\right)$ where:

(i) $\mathcal{O}=\operatorname{CAR}(\mathfrak{h})$ is the CAR algebra over the single particle Hilbert space $\mathfrak{h}$;

(ii) $\tau_{0}^{t}$ is the group of Bogoliubov $*$-automorphisms generated by the single particle Hamiltonian $h_{0}$,

$$
\tau_{0}^{t}\left(a^{\#}(f)\right)=a^{\#}\left(\mathrm{e}^{\mathrm{i} t h_{0}} f\right),
$$

where $a^{*}(f) / a(f)$ are the Fermi creation/annihilation operators associated to $f \in \mathfrak{h}$ and $a^{\#}$ stands for either $a$ or $a^{*}$. We denote by $\delta_{0}$ the generator of $\tau_{0}$.

Let $\mathfrak{O}$ be the $\tau_{0}$-invariant $C^{*}$-subalgebra of $\mathcal{O}$ generated by $\left\{a^{*}(f) a(g) \mid f, g \in \mathfrak{h}\right\}$ and $\mathbb{1}$. Physical observables are gauge invariant and hence the elements of $\mathfrak{O}$.

Let $\mathfrak{v}$ be a vector subspace of $\mathfrak{h}$ and let $\mathfrak{O}(\mathfrak{v})$ be the collection of the elements of the form

$$
A=\sum_{k=1}^{K} \prod_{j=1}^{n_{k}} a^{*}\left(f_{k j}\right) a\left(g_{k j}\right),
$$

where $K$ and $n_{k}$ 's are finite and $f_{k j}, g_{k j} \in \mathfrak{v}$. We denote $\bar{n}_{A} \equiv \max _{k} n_{k}$ and

$$
\mathcal{F}(A) \equiv\left\{f_{k j}, g_{k j} \mid j=1, \ldots, n_{k}, k=1, \ldots, K\right\}
$$

(to indicate the dependence of $K$ on $A$ we will also denote it by $\left.K_{A}\right) . \mathfrak{O}(\mathfrak{v})$ is a $*$-subalgebra of $\mathfrak{O}$, and if $\mathfrak{v}$ is dense in $\mathfrak{h}$, then $\mathfrak{O}(\mathfrak{v})$ is norm dense in $\mathfrak{O}$.

Our main assumption is :

(A) There exists a dense vector subspace $\mathfrak{d} \subset \mathfrak{h}$ such that the functions

$$
\mathbb{R} \ni t \mapsto\left(f, \mathrm{e}^{\mathrm{i} t h_{0}} g\right),
$$

are in $L^{1}(\mathbb{R}, \mathrm{d} t)$ for all $f, g \in \mathfrak{d}$.

This assumption implies that $h_{0}$ has purely absolutely continuous spectrum. Specific physical models which satisfy this assumption are discussed at the end of this subsection.

Let $V \in \mathfrak{O}(\mathfrak{d})_{\text {self }}$ be a self-adjoint perturbation. We shall always assume that $\bar{n}_{V} \geq 2$. The special case $\bar{n}_{V}=1$ leads to quasi-free perturbed dynamics and is discussed in detail in the companion paper [AJPP3], see also [AJPP1, AJPP2, JKP] and Remark after Theorem 1.7 below.

Let $\lambda \in \mathbb{R}$ be a coupling constant and let $\tau_{\lambda}$ be the $C^{*}$-dynamics generated by $\delta_{\lambda}=\delta_{0}+\mathrm{i} \lambda[V, \cdot]$. By rescaling $\lambda$, without loss of generality we may assume that

$$
\max _{f \in \mathcal{F}(V)}\|f\|=1 \text {. }
$$

We shall consider the locally interacting fermionic system described by $\left(\mathcal{O}, \tau_{\lambda}\right)$. Note that $\tau_{\lambda}$ preserves $\mathfrak{O}$ and that the pair $\left(\mathfrak{O}, \tau_{\lambda}\right)$ is also a $C^{*}$-dynamical system. Let

$$
\lambda_{V} \equiv \frac{1}{2 \bar{n}_{V} K_{V} \ell_{V}} \frac{\left(2 \bar{n}_{V}-2\right)^{2 \bar{n}_{V}-2}}{\left(2 \bar{n}_{V}-1\right)^{2 \bar{n}_{V}-1}}
$$

where

$$
\ell_{V} \equiv \int_{-\infty}^{\infty} \sup _{f, g \in \mathcal{F}(V)}\left|\left(f, \mathrm{e}^{\mathrm{i} t h_{0}} g\right)\right| \mathrm{d} t
$$

The following result was proven in [JOP4] (see also [BM1, AM, BM2, FMU]). 
Theorem 1.6 Suppose that (A) holds. Then:

1. For all $A \in \mathfrak{O}(\mathfrak{d})$ and any monomial $B=a^{\#}\left(f_{1}\right) \cdots a^{\#}\left(f_{m}\right)$ with $\left\{f_{1}, \ldots, f_{m}\right\} \subset \mathfrak{d}$, one has

$$
\sup _{|\lambda| \leq \lambda_{V}} \int_{\mathbb{R}}\left\|\left[\tau_{\lambda}^{t}(A), B\right]\right\| \mathrm{d} t<\infty
$$

2. For $|\lambda| \leq \lambda_{V}$ the Møller morphisms

$$
\gamma_{\lambda}^{+} \equiv \mathrm{s}-\lim _{t \rightarrow \infty} \tau_{0}^{-t} \circ \tau_{\lambda}^{t}
$$

exist and are $*$-automorphisms of $\mathcal{O}$.

In what follows we shall assume that (A) holds. Let $T$ be a self-adjoint operator on $\mathfrak{h}$ satisfying $0 \leq T \leq I$ and $\left[T\right.$, $\left.\mathrm{e}^{\mathrm{i} t h_{0}}\right]=0$ for all $t$, and let $\omega_{0}$ be the gauge invariant quasi-free state on $\mathcal{O}$ associated to $T$. We will sometimes call $T$ the density operator. The state $\omega_{0}$ is $\tau_{0}$-invariant and is the initial (reference) state of our fermionic system. The quantum dynamical system $\left(\mathcal{O}, \tau_{0}, \omega_{0}\right)$ is mixing. We denote by $\mathcal{N}_{0}$ the set of all $\omega$-normal states on $\mathcal{O}$. Theorem 1.6 yields that any state $\eta \in \mathcal{N}_{0}$ evolves to the limiting state $\omega_{\lambda}^{+}=\omega_{0} \circ \gamma_{\lambda}^{+}$, i.e., for $A \in \mathcal{O}$ and $|\lambda| \leq \lambda_{V}$,

$$
\lim _{t \rightarrow \infty} \eta\left(\tau_{\lambda}^{t}(A)\right)=\omega_{\lambda}^{+}(A)
$$

see, e.g., [Ro, AJPP1]. The state $\omega_{\lambda}^{+}$is the NESS (non-equilibrium steady state) of $\left(\mathcal{O}, \tau_{\lambda}\right)$ associated to the initial state $\omega_{0}$. Clearly, $\omega_{\lambda}^{+}$is $\tau_{\lambda}$-invariant and $\gamma_{\lambda}^{+}$is an isomorphism of the quantum dynamical systems $\left(\mathcal{O}, \tau_{0}, \omega_{0}\right)$ and $\left(\mathcal{O}, \tau_{\lambda}, \omega_{\lambda}^{+}\right)$. In particular, the system $\left(\mathcal{O}, \tau_{\lambda}, \omega_{\lambda}^{+}\right)$is mixing.

In what follows we shall always assume that $\operatorname{Ker} T=\operatorname{Ker}(I-T)=\{0\}$. This assumption ensures that the states $\omega_{0}$ and $\omega_{\lambda}^{+}$ are modular.

Let $\mathfrak{c} \subset \mathfrak{d}$ be a vector subspace such that the functions

$$
\mathbb{R} \ni t \mapsto\left(f, \mathrm{e}^{\mathrm{i} t h_{0}} T g\right),
$$

are in $L^{1}(\mathbb{R}, d t)$ for all $f, g \in \mathfrak{c}$. In general, it may happen that $\mathfrak{c}=\{0\}$, and so the existence of a non-trivial $\mathfrak{c}$ is a dynamical regularity property of the pair $\left(T, h_{0}\right)$. If $T=F\left(h_{0}\right)$, where $F \in L^{1}(\mathbb{R}, \mathrm{d} x)$ is such that its Fourier transform

$$
\hat{F}(t)=\frac{1}{\sqrt{2 \pi}} \int_{-\infty}^{\infty} \mathrm{e}^{\mathrm{i} t x} F(x) \mathrm{d} x
$$

is also in $L^{1}(\mathbb{R}, \mathrm{d} t)$, then one can take $\mathfrak{c}=\mathfrak{d}$.

Let

$$
\tilde{\lambda}_{V} \equiv 2^{-8\left(\bar{n}_{V}-1\right)} \lambda_{V}
$$

and

$$
\mathfrak{C} \equiv \mathfrak{O}(\mathfrak{c})
$$

The main result of this paper is:

Theorem 1.7 Suppose that (A) holds, that $V \in \mathfrak{C}_{\text {self }}$, and that $|\lambda| \leq \tilde{\lambda}_{V}$. Then $\mathfrak{C}$ is CLT-admissible and the QCLT holds for $\mathfrak{C}$ w.r.t. $\left(\mathcal{O}, \tau_{\lambda}, \omega_{\lambda}^{+}\right)$.

Remark. If $n_{V}=1$, then Theorem 1.6 holds for any $0<\lambda_{V}<\left(2 K_{V} \ell_{V}\right)^{-1}$, see [JOP4]. With this change, Theorem 1.7 holds with $\tilde{\lambda}_{V}=\lambda_{V}$. The case $n_{V}=1$ is however very special. If $V=\sum_{k} a^{*}\left(f_{k}\right) a\left(g_{k}\right)$, then $\tau_{\lambda}$ is quasi-free dynamics generated by $h_{\lambda}=h_{0}+\lambda \sum_{k}\left(g_{k}, \cdot\right) f_{k}$ and Theorem 1.6 can be derived from the scattering theory of the pair $\left(h_{\lambda}, h_{0}\right)$, see [Ro, AJPP1]. This alternative approach is technically simpler, yields better constants, and can be also used to prove a Large Deviation Principle and to discuss additional topics like Landauer-Büttiker formula which cannot be handled by the method of [JOP4] and this paper. For this reason, we shall discuss this special case separately in the companion paper [AJPP3].

As we have already remarked, our proof of Theorem 1.7 also yields the convergence of moments (see Theorem 3.2), and is easily extended to the proof of existence of QHL for locally interacting fermionic systems (recall (1.10), (1.11)). 
We finish this subsection with some concrete models to which Theorem 1.7 applies. The models on graphs are the same as in [JOP4]. Let $\mathcal{G}$ be the set of vertices of a connected graph of bounded degree, $\Delta_{\mathcal{G}}$ the discrete Laplacian acting on $l^{2}(\mathcal{G})$, and $\delta_{x}$ the Kronecker delta function at $x \in \mathcal{G}$. We shall call a graph $\mathcal{G}$ admissible if there exists $\gamma>1$ such that for all $x, y \in \mathcal{G}$,

$$
\left|\left(\delta_{x}, \mathrm{e}^{-\mathrm{i} t \Delta_{\mathcal{G}}} \delta_{y}\right)\right|=O\left(|t|^{-\gamma}\right),
$$

as $t \rightarrow \infty$. Examples of admissible graphs are $\mathcal{G}=\mathbb{Z}^{d}$ for $d \geq 3, \mathcal{G}=\mathbb{Z}_{+} \times \mathbb{Z}^{d-1}$ where $\mathbb{Z}_{+}=\{0,1, \cdots\}$ and $d \geq 1$, tubular graphs of the type $\mathbb{Z}_{+} \times \Gamma$, where $\Gamma \subset \mathbb{Z}^{d-1}$ is finite, a rooted Bethe lattice, etc. Assumption (A) holds and Theorem 1.7 holds with $\mathfrak{c}=\mathfrak{d}$ if:

(i) $\mathcal{G}$ is an admissible graph;

(ii) $\mathfrak{h}=\ell^{2}(\mathcal{G})$ (or more generally $\ell^{2}(\mathcal{G}) \otimes \mathbb{C}^{L}$ ) and $h_{0}=-\Delta_{\mathcal{G}}$;

(iii) $\mathfrak{d}$ is the subspace of finitely supported elements of $\mathfrak{h}$;

(iv) $T=F\left(h_{0}\right)$ where $\hat{F} \in L^{1}(\mathbb{R}, \mathrm{d} t)$ and $0<F(x)<1$ for $x \in \operatorname{sp}\left(h_{0}\right)$;

The continuous examples are similar. Let $\mathcal{D} \subset \mathbb{R}^{d}$ be a domain and let $\Delta_{\mathcal{D}}$ be the Dirichlet Laplacian on $L^{2}(\mathcal{D}, \mathrm{d} x)$. We shall say that a domain $\mathcal{D}$ is admissible if there exists $\gamma>1$ such that

$$
\left|\left(f, \mathrm{e}^{-\mathrm{i} t \Delta_{\mathcal{D}}} g\right)\right|=O\left(|t|^{-\gamma}\right),
$$

for all bounded $f$ and $g$ with compact support. Examples of admissible domains are $\mathcal{D}=\mathbb{R}^{d}$ for $d \geq 3, \mathcal{D}=\mathbb{R}_{+} \times \mathbb{R}^{d-1}$ for $d \geq 1$, tubular domains of the type $\mathbb{R}_{+} \times \Gamma$, where $\Gamma \subset \mathbb{R}^{d-1}$ is a bounded domain, etc. Assumption (A) holds and Theorem 1.7 holds with $\mathfrak{c}=\mathfrak{d}$ if:

(i) $\mathcal{D}$ is an admissible domain;

(ii) $\mathfrak{h}=L^{2}(\mathcal{D}, \mathrm{d} x)$ (or more generally $L^{2}(\mathcal{D}, \mathrm{d} x) \otimes \mathbb{C}^{L}$ ) and $h_{0}=-\Delta_{\mathcal{D}}$;

(iii) $\mathfrak{d}$ is the subspace of bounded compactly supported elements of $\mathfrak{h}$;

(iv) $T=F\left(h_{0}\right)$ where $\hat{F} \in L^{1}(\mathbb{R}, \mathrm{d} t)$ and $0<F(x)<1$ for $x \in \operatorname{sp}\left(h_{0}\right)$;

\subsection{QCLT, linear response and the Fluctuation-Dissipation theorem}

In addition to the assumptions of the previous subsection, we assume that $\mathfrak{h}, h_{0}, T$ have the composite structure

$$
\mathfrak{h}=\bigoplus_{j=1}^{M} \mathfrak{h}_{j}, \quad h_{0}=\bigoplus_{j=1}^{M} h_{j}, \quad T=\bigoplus_{j=1}^{M} \frac{1}{1+\mathrm{e}^{\beta_{j}\left(h_{j}-\mu_{j}\right)}},
$$

where $h_{j}$ 's are bounded from below self-adjoint operators on the Hilbert subspaces $\mathfrak{h}_{j}, \beta_{j}>0$, and $\mu_{j} \in \mathbb{R}$. We denote by $p_{j}$ the orthogonal projections onto $\mathfrak{h}_{j}$. The subalgebras $\mathcal{O}_{j}=\operatorname{CAR}\left(\mathfrak{h}_{j}\right)$ describe Fermi gas reservoirs $\mathcal{R}_{j}$ which are initially in equilibrium at inverse temperatures $\beta_{j}$ and chemical potentials $\mu_{j}$. The perturbation $\lambda V$ describes the interaction between the reservoirs and allows for the flow of heat and charges within the system.

The non-equilibrium statistical mechanics of this class of models has been studied recently in [JOP4] (see also [FMU] for related models and results). We briefly recall the results we need.

Suppose that $p_{j} \mathcal{F}(V) \subset \operatorname{Dom}\left(h_{j}\right)$ for all $j$. The entropy production observable of $\left(\mathcal{O}, \tau_{\lambda}\right)$ associated to the reference state $\omega_{0}$ is

$$
\sigma_{\lambda} \equiv-\sum_{j=1}^{M} \beta_{j}\left(\Phi_{j}-\mu_{j} \mathcal{J}_{j}\right)
$$

where $\Phi_{j} \equiv \mathrm{i} \lambda\left[\mathrm{d} \Gamma\left(h_{j} p_{j}\right), V\right]$ and $\mathcal{J}_{j} \equiv \mathrm{i} \lambda\left[\mathrm{d} \Gamma\left(p_{j}\right), V\right]$. Explicitly,

$$
\begin{gathered}
\Phi_{j}=\lambda \sum_{k=1}^{K_{V}} \sum_{l=1}^{n_{k}}\left(\prod_{i=1}^{l-1} a^{*}\left(f_{k i}\right) a\left(g_{k i}\right)\right)\left\{a^{*}\left(\mathrm{i} h_{j} p_{j} f_{k l}\right) a\left(g_{k l}\right)+a^{*}\left(f_{k l}\right) a\left(\mathrm{i} h_{j} p_{j} g_{k l}\right)\right\}\left(\prod_{i=l+1}^{n_{k}} a^{*}\left(f_{k i}\right) a\left(g_{k i}\right)\right), \\
\mathcal{J}_{j}=\lambda \sum_{k=1}^{K_{V}} \sum_{l=1}^{n_{k}}\left(\prod_{i=1}^{l-1} a^{*}\left(f_{k i}\right) a\left(g_{k i}\right)\right)\left\{a^{*}\left(\mathrm{i} p_{j} f_{k l}\right) a\left(g_{k l}\right)+a^{*}\left(f_{k l}\right) a\left(\mathrm{i} p_{j} g_{k l}\right)\right\}\left(\prod_{i=l+1}^{n_{k}} a^{*}\left(f_{k i}\right) a\left(g_{k i}\right)\right) .
\end{gathered}
$$


The observable $\Phi_{j} / \mathcal{J}_{j}$ describes the heat/charge flux out of the reservoir $\mathcal{R}_{j}$ (note that $\Phi_{j}, \mathcal{J}_{j} \in \mathfrak{O}$ ). The conservation laws

$$
\sum_{j=1}^{M} \omega_{\lambda}^{+}\left(\Phi_{j}\right)=0, \quad \sum_{j=1}^{M} \omega_{\lambda}^{+}\left(\mathcal{J}_{j}\right)=0
$$

hold. By the general result of [JP1, Ru2, JP4], the entropy production of the NESS $\omega_{\lambda}^{+}$is non-negative,

$$
\operatorname{Ep}\left(\omega_{\lambda}^{+}\right) \equiv \omega_{\lambda}^{+}\left(\sigma_{\lambda}\right)=-\sum_{j=1}^{M} \beta_{j}\left(\omega_{\lambda}^{+}\left(\Phi_{j}\right)-\mu_{j} \omega_{\lambda}^{+}\left(\mathcal{J}_{j}\right)\right) \geq 0 .
$$

If all $\beta_{j}$ 's and $\mu_{j}$ 's are equal, i.e. $\beta_{1}=\cdots=\beta_{M}=\beta$ and $\mu_{1}=\cdots=\mu_{M}=\mu$, then $\omega_{0}\left\lceil\mathfrak{O}\right.$ is a $\left(\tau_{0}, \beta\right)$-KMS state and so the reference state is a thermal equilibrium state of the unperturbed system. Then $\omega_{\lambda}^{+} \uparrow \mathfrak{O}$ is a $\left(\tau_{\lambda}, \beta\right)$-KMS state, $\omega_{\lambda}^{+}\left(\Phi_{j}\right)=\omega_{\lambda}^{+}\left(\mathcal{J}_{j}\right)=0$ for all $j$, and in particular $\operatorname{Ep}\left(\omega_{\lambda}^{+}\right)=0$, see [JOP2]. On physical grounds, vanishing of the fluxes and the entropy production in thermal equilibrium is certainly an expected result. It is also expected that if either $\beta_{j}$ 's or $\mu_{j}$ 's are not all equal, then $\operatorname{Ep}\left(\omega_{\lambda}^{+}\right)>0$. For specific interactions $V$ one can compute $\omega_{\lambda}^{+}\left(\sigma_{\lambda}\right)$ to the first non-trivial order in $\lambda$ and hence establish the strict positivity of entropy production by a perturbative calculation (see [FMU, JP6] and [JP3] for a related results). The strict positivity of the entropy production for a generic perturbation $\lambda V$ has been established in [JP5].

To establish QCLT for the flux observables in addition to the Assumption (A) we need:

(B) For all $j, h_{j} p_{j} \mathfrak{d} \subset \mathfrak{d}$.

This assumption and the specific form of density operator ensure that one may take $\mathfrak{c}=\mathfrak{d}$ and that if $V \in \mathfrak{C}_{\text {self }}$, then $\left\{\Phi_{j}, \mathcal{J}_{j}\right\} \subset \mathfrak{C}_{\text {self }}$. Hence, for $|\lambda| \leq \tilde{\lambda}_{V}$ the QCLT holds for the flux observables.

We finish with a discussion of linear response theory (for references and additional information about linear response theory in algebraic formalism of quantum statistical mechanics we refer the reader to [AJPP1] and [JOP1]-[JOP4]). We will need the following two assumptions:

(C) The operators $h_{j}$ are bounded.

(D) There exists a complex conjugation $c$ on $\mathfrak{h}$ which commutes with all $h_{j}$ and satisfies $c f=f$ for all $f \in \mathcal{F}(V)$.

Assumption (C) is of technical nature and can be relaxed. Assumption (D) ensures that the system $\left(\mathcal{O}, \tau_{\lambda}, \omega_{0}\right)$ is time-reversal invariant. Time-reversal invariance is of central importance in linear response theory.

Let $\beta_{\text {eq }}>0$ and $\mu_{\text {eq }} \in \mathbb{R}$ be given equilibrium values of the inverse temperature and chemical potential. We denote $\vec{\beta}=$ $\left(\beta_{1}, \cdots, \beta_{M}\right), \vec{\mu}=\left(\mu_{1}, \cdots, \mu_{M}\right), \vec{\beta}_{\mathrm{eq}}=\left(\beta_{\mathrm{eq}}, \cdots, \beta_{\mathrm{eq}}\right), \vec{\mu}_{\mathrm{eq}}=\left(\mu_{\mathrm{eq}}, \cdots, \mu_{\mathrm{eq}}\right)$, and we shall indicate explicitly the dependence of $\omega_{\lambda}^{+}$on $\vec{\beta}$ and $\vec{\mu}$ by $\omega_{\lambda, \vec{\beta}, \vec{\mu}}^{+}$. Similarly, we shall indicate explicitly the dependence of $L(A, B)$ on $\lambda, \vec{\beta}, \vec{\mu}$ by $L_{\lambda, \vec{\beta}, \vec{\mu}}$. Since $\omega_{\lambda, \vec{\beta}_{\mathrm{eq}}, \vec{\mu}_{\mathrm{eq}}}^{+}\left(\Phi_{j}\right)=\omega_{\lambda, \vec{\beta}_{\mathrm{eq}}, \vec{\mu}_{\mathrm{eq}}}^{+}\left(\mathcal{J}_{j}\right)=0$,

$$
L_{\lambda, \vec{\beta}_{\mathrm{eq}}, \vec{\mu}_{\mathrm{eq}}}(A, B)=\int_{-\infty}^{\infty} \omega_{\lambda, \vec{\beta}_{\mathrm{eq}}, \vec{\mu}_{\mathrm{eq}}}^{+}\left(A \tau_{\lambda}^{t}(B)\right) \mathrm{d} t
$$

for $A, B \in\left\{\Phi_{j}, \mathcal{J}_{j} \mid 1 \leq j \leq M\right\}$.

Assuming the existence of derivatives, the kinetic transport coefficients are defined by

$$
\begin{array}{ll}
\mathcal{L}_{\lambda \mathrm{hh}}^{k j} \equiv-\left.\partial_{\beta_{j}} \omega_{\lambda, \vec{\beta}, \vec{\mu}}^{+}\left(\Phi_{k}\right)\right|_{\vec{\beta} \equiv \vec{\beta}_{\mathrm{eq}}, \vec{\mu}=\vec{\mu}_{\mathrm{eq}}}, & \left.\mathcal{L}_{\lambda \mathrm{hc}}^{k j} \equiv \beta_{\mathrm{eq}} \partial_{\mu_{j}} \omega_{\lambda, \vec{\beta}, \vec{\mu}}^{+}\left(\Phi_{k}\right)\right|_{\vec{\beta}=\vec{\beta}_{\mathrm{eq}}, \vec{\mu}=\vec{\mu}_{\mathrm{eq}}}, \\
\mathcal{L}_{\lambda \mathrm{ch}}^{k j} \equiv-\left.\partial_{\beta_{j}} \omega_{\lambda, \vec{\beta}, \vec{\mu}}^{+}\left(\mathcal{J}_{k}\right)\right|_{\vec{\beta}=\vec{\beta}_{\mathrm{eq}}, \vec{\mu}=\vec{\mu}_{\mathrm{eq}}}, & \left.\mathcal{L}_{\lambda \mathrm{cc}}^{k j} \equiv \beta_{\mathrm{eq}} \partial_{\mu_{j}} \omega_{\lambda, \vec{\beta}, \vec{\mu}}^{+}\left(\mathcal{J}_{k}\right)\right|_{\vec{\beta}=\vec{\beta}_{\mathrm{eq}}, \vec{\mu}=\vec{\mu}_{\mathrm{eq}}},
\end{array}
$$

where the indices $\mathrm{h} / \mathrm{c}$ stand for heat/charge. We then have 
Theorem 1.8 Suppose that Assumptions (A)-(D) hold. Then, for any $|\lambda|<\lambda_{V}$, the functions

$$
(\vec{\beta}, \vec{\mu}) \mapsto \omega_{\lambda, \vec{\beta}, \vec{\mu}}^{+}\left(\Phi_{j}\right), \quad(\vec{\beta}, \vec{\mu}) \mapsto \omega_{\lambda, \vec{\beta}, \vec{\mu}}^{+}\left(\mathcal{J}_{j}\right),
$$

are analytic in a neighborhood of $\left(\vec{\beta}_{\mathrm{eq}}, \vec{\mu}_{\mathrm{eq}}\right)$. Moreover,

(1) The Green-Kubo formulas hold:

$$
\begin{array}{ll}
\mathcal{L}_{\lambda \mathrm{hh}}^{k j}=\frac{1}{2} L_{\lambda, \vec{\beta}_{\mathrm{eq}}, \vec{\mu}_{\mathrm{eq}}}\left(\Phi_{k}, \Phi_{j}\right), & \mathcal{L}_{\lambda \mathrm{hc}}^{k j}=\frac{1}{2} L_{\lambda, \vec{\beta}_{\mathrm{eq}}, \vec{\mu}_{\mathrm{eq}}}\left(\Phi_{k}, \mathcal{J}_{j}\right), \\
\mathcal{L}_{\lambda \mathrm{ch}}^{k j}=\frac{1}{2} L_{\lambda, \vec{\beta}_{\mathrm{eq}}, \vec{\mu}_{\mathrm{eq}}}\left(\mathcal{J}_{k}, \Phi_{j}\right), & \mathcal{L}_{\lambda \mathrm{cc}}^{k j}=\frac{1}{2} L_{\lambda, \vec{\beta}_{\mathrm{eq}}, \vec{\mu}_{\mathrm{eq}}}\left(\mathcal{J}_{k}, \mathcal{J}_{j}\right) .
\end{array}
$$

(2) The Onsager reciprocity relations hold:

$$
\mathcal{L}_{\lambda \mathrm{hh}}^{k j}=\mathcal{L}_{\lambda \mathrm{hh}}^{j k}, \quad \mathcal{L}_{\lambda \mathrm{cc}}^{k j}=\mathcal{L}_{\lambda \mathrm{cc}}^{j k}, \quad \mathcal{L}_{\lambda \mathrm{hc}}^{k j}=\mathcal{L}_{\lambda \mathrm{ch}}^{j k} .
$$

(3) Let $\mathfrak{C}$ denote the linear span of $\left\{\Phi_{j}, \mathcal{J}_{j} \mid 1 \leq j \leq M\right\}$. For $|\lambda| \leq \tilde{\lambda}_{V}$, $\mathfrak{C}$ is CLT-admissible and the QCLT holds for $\mathfrak{C}$ w.r.t. $\left(\mathcal{O}, \tau_{\lambda}, \omega_{\lambda, \vec{\beta}_{\mathrm{eq}}, \vec{\mu}_{\mathrm{eq}}}\right)$. The associated fluctuation algebra $\mathcal{W}$ is commutative.

Remark 1. Parts (1) and (2) of Theorem 1.8 are proven in [JOP4]. Part (3) is a special case of Theorem 1.7. Parts (1) and (3) relate linear response to thermodynamical forces to fluctuations in thermal equilibrium and constitute the FluctuationDissipation Theorem for our model. The physical aspects of linear response theory and Fluctuation-Dissipation Theorem are discussed in classical references [DGM, KTH].

Remark 2. The arguments in [JOP4] do not establish that the functions

$$
t \mapsto \omega_{\lambda, \vec{\beta}_{\mathrm{eq}}, \vec{\mu}_{\mathrm{eq}}}^{+}\left(A \tau_{\lambda}^{t}(B)\right)
$$

are absolutely integrable for $A, B \in\left\{\Phi_{j}, \mathcal{J}_{j} \mid 1 \leq j \leq M\right\}$ and in Part (2) $L_{\lambda, \vec{\beta}_{\text {eq }}, \vec{\mu}_{\text {eq }}}(A, B)$ is defined by

$$
L_{\lambda, \vec{\beta}_{\mathrm{eq}}, \vec{\mu}_{\mathrm{eq}}}(A, B)=\lim _{t \rightarrow \infty} \int_{-t}^{t} \omega_{\lambda, \vec{\beta}_{\mathrm{eq}}, \vec{\mu}_{\mathrm{eq}}}^{+}\left(A \tau_{\lambda}^{s}(B)\right) \mathrm{d} s
$$

The absolute integrability of the correlation functions (1.23) is a delicate dynamical problem resolved in Part (3) for $|\lambda| \leq \tilde{\lambda}_{V}$ (see Definition 1.1).

Remark 3. Remarks 4 and 6 after Theorem 1.5 in [JOP4] apply without changes to Theorem 1.8. Remark 7 is also applicable and allows to extend the Fluctuation-Dissipation Theorem to a large class of so called centered observables.

Remark 4. Although the time-reversal Assumption (D) plays no role in Part (3) of Theorem 1.8, it is a crucial ingredient in proofs of Parts (1) and (2) (see [JOP4, AJPP3] for a discussion). The Fluctuation-Dissipation Theorem fails for locally interacting open fermionic systems which are not time-reversal invariant.

A class of concrete models for which (A)-(B)-(D) hold is easily constructed following the examples discussed at the end of Subsection 1.2. Let $\mathcal{G}_{1}, \ldots, \mathcal{G}_{M}$ be admissible graphs. Then (A)-(D) hold if $\mathfrak{h}_{j}=\ell^{2}\left(\mathcal{G}_{j}\right)\left(\right.$ or $\left.\ell^{2}\left(\mathcal{G}_{j}\right) \otimes \mathbb{C}^{L}\right), h_{j}=-\Delta_{\mathcal{G}_{j}}$, and $\mathfrak{d}$ is the subspace of finitely supported elements of $\mathfrak{h}$. A physically important class of allowed interactions is $V=V^{\text {hop }}+V^{\text {int }}$ where

$$
V^{\mathrm{hop}}=\sum_{x, y} t(x, y)\left(a^{*}\left(\delta_{x}\right) a\left(\delta_{y}\right)+a^{*}\left(\delta_{y}\right) a\left(\delta_{x}\right)\right),
$$

and $t: \mathcal{G} \times \mathcal{G} \rightarrow \mathbb{R}$ is a finitely supported function $\left(\mathcal{G}=\cup_{j} \mathcal{G}_{j}\right)$, and

$$
V^{\mathrm{int}}=\sum_{x, y} v(x, y) a^{*}\left(\delta_{x}\right) a^{*}\left(\delta_{y}\right) a\left(\delta_{y}\right) a\left(\delta_{x}\right)
$$

where $v: \mathcal{G} \times \mathcal{G} \rightarrow \mathbb{R}$ is finitely supported. $V^{\text {hop }}$ describes tunneling junctions between the reservoir and $V^{\text {int }}$ is a local pair interaction. 


\section{General aspects of CLT}

\subsection{Proof of Theorem 1.4}

Our argument follows the ideas of $[\mathrm{GV}]$. For $A, B$ in $\mathcal{O}_{\text {self }}$ we set

$$
D(A, B) \equiv \mathrm{e}^{\mathrm{i} A} \mathrm{e}^{\mathrm{i} B}-\mathrm{e}^{\mathrm{i}(A+B)} \mathrm{e}^{-\frac{1}{2}[A, B]} .
$$

The first ingredient of the proof is:

Proposition 2.1 If the set $\{A, B\} \subset \mathcal{O}_{\text {self }}$ is $L^{1}$-asymptotically abelian for $\tau$ then the asymptotic 2 nd-order Baker-CampbellHausdorff formula

$$
\lim _{t \rightarrow \infty}\left\|D\left(\tilde{A}_{t}, \tilde{B}_{t}\right)\right\|=0,
$$

holds.

Note that Proposition 2.1 is not a simple consequence of the $\mathrm{BCH}$ formula because its hypothesis do not ensure that the double commutator $\left[\tilde{A}_{t},\left[\tilde{A}_{t}, \tilde{B}_{t}\right]\right]$ vanishes as $t \rightarrow \infty$. To prove Proposition 2.1 we need the following estimate.

Lemma 2.2 If $A, B, a, b$ are bounded self-adjoint operators then

$$
\|D(A+a, B+b)\| \leq\|D(A, B)\|+4\left(\|a\|^{3}+\|b\|^{3}\right)+\|[[A, B],[a, b]]\|+(2+\|a\|+\|b\|) \sum_{\substack{x \in\{A, B\} \\ y \in\{a, b\}}}\|[X, y]\| .
$$

Proof. We decompose $D(A+a, B+b)=\sum_{j=1}^{9} D_{j}$ according to the following table and get an upper bound of the norm of each term using the elementary estimates

$$
\left\|\mathrm{e}^{\mathrm{i}(x+y)}-\mathrm{e}^{\mathrm{i} x}\right\| \leq\|y\|, \quad\left\|\mathrm{e}^{\mathrm{i}(x+y)}-\mathrm{e}^{\mathrm{i} x} \mathrm{e}^{\mathrm{i} y}\right\| \leq \frac{1}{2}\|[x, y]\|, \quad\left\|\mathrm{e}^{\mathrm{i} x} \mathrm{e}^{\mathrm{i} y}-\mathrm{e}^{\mathrm{i} y} \mathrm{e}^{\mathrm{i} x}\right\| \leq\|[x, y]\| .
$$

\begin{tabular}{|l|l|l|}
\hline$j$ & \multicolumn{1}{|c|}{$D_{j}$} & \multicolumn{1}{|c|}{ upper bound on $\left\|D_{j}\right\|$} \\
\hline \hline 1 & $\left(\mathrm{e}^{\mathrm{i}(A+a)}-\mathrm{e}^{\mathrm{i} a} \mathrm{e}^{\mathrm{i} A}\right) \mathrm{e}^{\mathrm{i}(B+b)}$ & $\frac{1}{2}\|[A, a]\|$ \\
\hline 2 & $\mathrm{e}^{\mathrm{i} a} \mathrm{e}^{\mathrm{i} A}\left(\mathrm{e}^{\mathrm{i}(B+b)}-\mathrm{e}^{\mathrm{i} b} \mathrm{e}^{\mathrm{i} B}\right)$ & $\frac{1}{2}\|[B, b]\|$ \\
\hline 3 & $\mathrm{e}^{\mathrm{i} a}\left(\mathrm{e}^{\mathrm{i} A} \mathrm{e}^{\mathrm{i} b}-\mathrm{e}^{\mathrm{i} b} \mathrm{e}^{\mathrm{i} A}\right) \mathrm{e}^{\mathrm{i} B}$ & $\|[A, b]\|$ \\
\hline 4 & $\mathrm{e}^{\mathrm{i} a} \mathrm{e}^{\mathrm{i} b}\left(\mathrm{e}^{\mathrm{i} A} \mathrm{e}^{\mathrm{i} B}-\mathrm{e}^{\mathrm{i}(A+B)} \mathrm{e}^{-\frac{1}{2}[A, B]}\right)$ & $\|D(A, B)\|$ \\
\hline 5 & $\left(\mathrm{e}^{\mathrm{i} a} \mathrm{e}^{\mathrm{i} b}-\mathrm{e}^{\mathrm{i}(a+b)} \mathrm{e}^{-\frac{1}{2}[a, b]}\right) \mathrm{e}^{\mathrm{i}(A+B)} \mathrm{e}^{-\frac{1}{2}[A, B]}$ & $\|D(a, b)\|$ \\
\hline 6 & $\mathrm{e}^{\mathrm{i}(a+b)}\left(\mathrm{e}^{-\frac{1}{2}[a, b]} \mathrm{e}^{\mathrm{i}(A+B)}-\mathrm{e}^{\mathrm{i}(A+B)} \mathrm{e}^{-\frac{1}{2}[a, b]}\right) \mathrm{e}^{-\frac{1}{2}[A, B]}$ & $\frac{1}{2}\|[A+B,[a, b]]\|$ \\
\hline 7 & $\mathrm{e}^{\mathrm{i}(a+b)} \mathrm{e}^{\mathrm{i}(A+B)}\left(\mathrm{e}^{-\frac{1}{2}[a, b]} \mathrm{e}^{-\frac{1}{2}[A, B]}-\mathrm{e}^{-\frac{1}{2}[A, B]-\frac{1}{2}[a, b]}\right)$ & $\frac{1}{8}\|[[A, B],[a, b]]\|$ \\
\hline 8 & $\mathrm{e}^{\mathrm{i}(a+b)} \mathrm{e}^{\mathrm{i}(A+B)}\left(\mathrm{e}^{-\frac{1}{2}[A, B]-\frac{1}{2}[a, b]}-\mathrm{e}^{-\frac{1}{2}[A+a, B+b]}\right)$ & $\frac{1}{2}(\|[A, b]\|+\|[B, a]\|)$ \\
\hline 9 & $\left(\mathrm{e}^{\mathrm{i}(a+b)} \mathrm{e}^{\mathrm{i}(A+B)}-\mathrm{e}^{\mathrm{i}(A+B+a+b)}\right) \mathrm{e}^{-\frac{1}{2}[A+a, B+b]}$ & $\frac{1}{2}(\|[A, a]\|+\|[A, b]\|+\|[B, a]\|+\|[B, b]\|)$ \\
\hline
\end{tabular}


$>$ From the $\mathrm{BCH}$ estimate we further get

$$
\left\|D_{5}\right\| \leq\|D(a, b)\| \leq\|[a,[a, b]]\|+\|[b,[a, b]]\| \leq 4\left(\|a\|^{3}+\|b\|^{3}\right),
$$

and the Jacobi identity yields

$$
\left\|D_{6}\right\| \leq\|a\|(\|[A, b]\|+\|[B, b]\|)+\|b\|(\|[A, a]\|+\|[B, a]\|) .
$$

The result follows.

Proof of Proposition 2.1. For $t>0$ and $j \in \mathbb{N}$ set $p(t) \equiv \log (1+t)$ and $I_{j}(t) \equiv\left[j p(t),(j+1) p(t)\left[\right.\right.$. For $X \in \mathcal{O}_{\text {self }}$ define

$$
X_{t}^{(j)} \equiv t^{-1 / 2} \int_{I_{j}(t) \cap[0, t]} \tau^{s}(X) \mathrm{d} s, \quad X_{t}^{(<k)} \equiv \sum_{0 \leq j<k} X_{t}^{(j)} .
$$

If $N(t)$ denotes the integer such that $N(t) p(t) \leq t<(N(t)+1) p(t)$ then repeated use of Lemma 2.2 yields

$$
\begin{aligned}
\left\|D\left(\tilde{A}_{t}, \tilde{B}_{t}\right)\right\| \leq & 4 \sum_{j=0}^{N(t)}\left(\left\|A_{t}^{(j)}\right\|^{3}+\left\|B_{t}^{(j)}\right\|^{3}\right)+\sum_{j=0}^{N(t)}\left(2+\left\|A_{t}^{(j)}\right\|+\left\|B_{t}^{(j)}\right\|\right) \sum_{X, Y \in\{A, B\}}\left\|\left[X_{t}^{(<j)}, Y_{t}^{(j)}\right]\right\| \\
& +\sum_{j=0}^{N(t)}\left\|\left[\left[A_{t}^{(<j)}, B_{t}^{(<j)}\right],\left[A_{t}^{(j)}, B_{t}^{(j)}\right]\right]\right\| .
\end{aligned}
$$

We now estimate the right hand side of this inequality. We first note that

$$
\left\|X_{t}^{(j)}\right\| \leq\|X\| t^{-1 / 2} p(t) \leq\|X\|
$$

and hence

$$
\sum_{j=0}^{N(t)}\left(\left\|A_{t}^{(j)}\right\|^{3}+\left\|B_{t}^{(j)}\right\|^{3}\right) \leq\left(\|A\|^{3}+\|B\|^{3}\right)(N(t)+1) t^{-3 / 2} p(t)^{3} \leq 2\left(\|A\|^{3}+\|B\|^{3}\right) t^{-1 / 2} p(t)^{2} \rightarrow 0,
$$

as $t \rightarrow \infty$. Next consider

$$
\sum_{j=0}^{N(t)} \sum_{k=0}^{j-1}\left\|\left[X_{t}^{(k)}, Y_{t}^{(j)}\right]\right\| \leq \frac{1}{t} \sum_{j=0}^{N(t)} \int_{0}^{j p(t)} \int_{j p(t)}^{(j+1) p(t)}\left\|\left[X, \tau^{v-u}(Y)\right]\right\| \mathrm{d} v \mathrm{~d} u
$$

The change of variables $\xi=v-j p(t), \eta=v-u$, leads to

$$
\sum_{j=0}^{N(t)} \sum_{k=0}^{j-1}\left\|\left[X_{t}^{(k)}, Y_{t}^{(j)}\right]\right\| \leq \frac{1}{t} \sum_{j=0}^{N(t)} \int_{0}^{p(t)} \int_{\xi}^{\xi+j p(t)}\left\|\left[X, \tau^{\eta}(Y)\right]\right\| \mathrm{d} \eta \mathrm{d} \xi \leq \frac{N(t)+1}{t} \int_{0}^{p(t)} \int_{\xi}^{\infty}\left\|\left[X, \tau^{\eta}(Y)\right]\right\| \mathrm{d} \eta \mathrm{d} \xi .
$$

Since $(N(t)+1) / t \leq 2 / p(t)$ we obtain, for $X, Y \in\{A, B\}$,

$$
\lim _{t \rightarrow \infty} \sum_{j=0}^{N(t)} \sum_{k=0}^{j-1}\left\|\left[X_{t}^{(k)}, Y_{t}^{(j)}\right]\right\| \leq \lim _{p \rightarrow \infty} \frac{2}{p} \int_{0}^{p}\left[\int_{\xi}^{\infty}\left\|\left[X, \tau^{\eta}(Y)\right]\right\| \mathrm{d} \eta\right] \mathrm{d} \xi=0 .
$$

Combining this with (2.25) we get

$$
\sum_{j=0}^{N(t)}\left(2+\left\|A_{t}^{(j)}\right\|+\left\|B_{t}^{(j)}\right\|\right) \sum_{X, Y \in\{A, B\}}\left\|\left[X_{t}^{(<j)}, Y_{t}^{(j)}\right]\right\| \leq(2+\|A\|+\|B\|) \sum_{X, Y \in\{A, B\}} \sum_{j=0}^{N(t)} \sum_{k=0}^{j-1}\left\|\left[X_{t}^{(k)}, Y_{t}^{(j)}\right]\right\| \rightarrow 0
$$

as $t \rightarrow \infty$. To estimate the last term on the right hand side of (2.24) we write

$$
\sum_{j=0}^{N(t)}\left\|\left[\left[A_{t}^{(<j)}, B_{t}^{(<j)}\right],\left[A_{t}^{(j)}, B_{t}^{(j)}\right]\right]\right\| \leq \sum_{j=0}^{N(t)} \sum_{k=0}^{j-1} \sum_{l=0}^{j-1}\left\|\left[\left[A_{t}^{(k)}, B_{t}^{(l)}\right],\left[A_{t}^{(j)}, B_{t}^{(j)}\right]\right]\right\|=Z_{1}+Z_{2}+Z_{3},
$$


where

$$
Z_{1} \equiv \sum_{j=0}^{N(t)} \sum_{k=0}^{j-1}\left\|\left[\left[A_{t}^{(k)}, B_{t}^{(k)}\right],\left[A_{t}^{(j)}, B_{t}^{(j)}\right]\right]\right\|
$$

and

$$
Z_{2} \equiv \sum_{j=0}^{N(t)} \sum_{k=0}^{j-1} \sum_{l=0}^{k-1}\left\|\left[\left[A_{t}^{(k)}, B_{t}^{(l)}\right],\left[A_{t}^{(j)}, B_{t}^{(j)}\right]\right]\right\|, \quad Z_{3} \equiv \sum_{j=0}^{N(t)} \sum_{l=0}^{j-1} \sum_{k=0}^{l-1}\left\|\left[\left[A_{t}^{(k)}, B_{t}^{(l)}\right],\left[A_{t}^{(j)}, B_{t}^{(j)}\right]\right]\right\| .
$$

Combined with (2.25) and (2.26), the identity

$$
\left[\left[A_{k}, B_{k}\right],\left[A_{j}, B_{j}\right]\right]=\left[\left[\left[A_{k}, A_{j}\right], B_{k}\right], B_{j}\right]+\left[\left[\left[A_{j}, B_{k}\right], A_{k}\right], B_{j}\right]+\left[\left[\left[B_{k}, B_{j}\right], A_{k}\right], A_{j}\right]+\left[\left[\left[B_{j}, A_{k}\right], B_{k}\right], A_{j}\right],
$$

yields

$$
Z_{1} \leq 4\left(\|A\|^{2}+\|B\|^{2}\right) \sum_{X, Y \in\{A, B\}} \sum_{j=0}^{N(t)} \sum_{k=0}^{j-1}\left\|\left[X_{t}^{(k)}, Y_{t}^{(j)}\right]\right\| \rightarrow 0
$$

as $t \rightarrow \infty$. The estimate

$$
\begin{aligned}
\sum_{j=0}^{N(t)}\left\|\left[A_{t}^{(j)}, B_{t}^{(j)}\right]\right\| & \leq \frac{1}{t} \sum_{j=0}^{N(t)} \int_{I_{j}(t)} \int_{I_{j}(t)}\left\|\left[A, \tau^{u-v}(B)\right]\right\| \mathrm{d} u \mathrm{~d} v \\
& \leq \frac{1}{t} \sum_{j=0}^{N(t)} \int_{0}^{p(t)} \int_{-v}^{p(t)-v}\left\|\left[A, \tau^{u}(B)\right]\right\| \mathrm{d} u \mathrm{~d} v \\
& \leq \frac{N(t)+1}{t} p(t) \int_{-p(t)}^{p(t)}\left\|\left[A, \tau^{u}(B)\right]\right\| \mathrm{d} u \leq 2 \int_{\mathbb{R}}\left\|\left[A, \tau^{u}(B)\right]\right\| \mathrm{d} u
\end{aligned}
$$

together with (2.26) yield

$$
Z_{2} \leq 2 \sum_{j=0}^{N(t)}\left\|\left[A_{t}^{(j)}, B_{t}^{(j)}\right]\right\| \sum_{k=0}^{N(t)} \sum_{l=0}^{k-1}\left\|\left[A_{t}^{(k)}, B_{t}^{(l)}\right]\right\| \rightarrow 0,
$$

as $t \rightarrow \infty$. The same argument applies to $Z_{3}$ and completes the proof. $\square$

Let $\left(\mathcal{H}_{\omega}, \pi_{\omega}, \Omega_{\omega}\right)$ be the GNS-representation of the algebra $\mathcal{O}$ associated to the state $\omega$. The second ingredient of the proof of Theorem 1.4 is:

Proposition 2.3 Suppose that $(\mathcal{O}, \tau, \omega)$ is an ergodic quantum dynamical system and that $\omega$ is a modular state. If $\{A, B\}$ is an $L^{1}$-asymptotically abelian pair for $\tau$, then

$$
\mathrm{s}-\lim _{t \rightarrow \infty} \pi_{\omega}\left(\left[\tilde{A}_{t}, \tilde{B}_{t}\right]\right)=\int_{-\infty}^{\infty} \omega\left(\left[\tau^{s}(A), B\right]\right) \mathrm{d} s .
$$

Proof. We shall first prove that

$$
\lim _{t \rightarrow \infty} \pi_{\omega}\left(\left[\tilde{A}_{t}, \tilde{B}_{t}\right]\right) \Omega_{\omega}=\left(\int_{-\infty}^{\infty} \omega\left(\left[\tau^{s}(A), B\right]\right) \mathrm{d} s\right) \Omega_{\omega}
$$

Writing

$$
\pi_{\omega}\left(\left[\tilde{A}_{t}, \tilde{B}_{t}\right]\right)=\frac{1}{t} \int_{0}^{t} \int_{0}^{t} \pi_{\omega}\left(\tau^{s_{1}}\left(\left[\tau^{s_{2}-s_{1}}(A), B\right]\right)\right) \mathrm{d} s_{1} \mathrm{~d} s_{2},
$$

the change of variable $u=s_{1}, v=s_{2}-s_{1}$ yields that

$$
\pi_{\omega}\left(\left[\tilde{A}_{t}, \tilde{B}_{t}\right]\right)=\int_{-t}^{t} f_{t}(v) \mathrm{d} v
$$


where

Clearly,

$$
f_{t}(v)=\frac{1}{t} \int_{\max (-v, 0)}^{\min (t, t-v)} \pi_{\omega}\left(\tau^{u}\left(\left[\tau^{v}(A), B\right]\right)\right) \mathrm{d} u
$$

$$
\left.\left\|f_{t}(v)\right\| \leq \|\left[\tau^{v}(A), B\right]\right) \| \in L^{1}(\mathbb{R})
$$

and so, by the dominated convergence theorem, it suffices to show that

$$
\lim _{t \rightarrow \infty} f_{t}(v) \Omega_{\omega}=\omega\left(\left[\tau^{v}(A), B\right]\right) \Omega_{\omega},
$$

for all $v \in \mathbb{R}$ to prove (2.27). Let $\mathcal{L}_{\omega}$ be the standard Liouvillean associated to $\omega$. We recall that $\mathcal{L}_{\omega}$ is the unique self-adjoint operator on $\mathcal{H}_{\omega}$ such that

$$
\pi_{\omega}\left(\tau^{t}(A)\right)=\mathrm{e}^{\mathrm{i} t \mathcal{L}_{\omega}} \pi_{\omega}(A) \mathrm{e}^{-\mathrm{i} t \mathcal{L}_{\omega}}, \quad \mathcal{L}_{\omega} \Omega_{\omega}=0 .
$$

Then

$$
\pi_{\omega}\left(\tau^{u}\left(\left[\tau^{v}(A), B\right]\right)\right) \Omega_{\omega}=\mathrm{e}^{\mathrm{i} u \mathcal{L}_{\omega}} \pi_{\omega}\left(\left[\tau^{v}(A), B\right]\right) \Omega_{\omega}
$$

implies

$$
f_{t}(v) \Omega_{\omega}=\frac{1}{t} \int_{\max (-v, 0)}^{\min (t, t-v)} \mathrm{e}^{\mathrm{i} u \mathcal{L}_{\omega}} \pi_{\omega}\left(\left[\tau^{v}(A), B\right]\right) \Omega_{\omega} \mathrm{d} u .
$$

Since $(\mathcal{O}, \tau, \omega)$ is ergodic, zero is a simple eigenvalue of $\mathcal{L}_{\omega}$, and von Neumann's mean ergodic theorem yields

$$
\mathrm{s}-\lim _{t \rightarrow \infty} \frac{1}{t} \int_{\max (-v, 0)}^{\min (t, t-v)} \mathrm{e}^{\mathrm{i} u \mathcal{L}_{\omega}} \mathrm{d} u=\mathrm{s}-\lim _{t \rightarrow \infty} \frac{1}{t} \int_{0}^{t} \mathrm{e}^{\mathrm{i} u \mathcal{L}_{\omega}} \mathrm{d} u=\Omega_{\omega}\left(\Omega_{\omega} \mid \cdot\right),
$$

for all $v \in \mathbb{R}$. This implies (2.30) and (2.27) follows.

To finish the proof note that for any $X \in \pi_{\omega}(\mathcal{O})^{\prime}$ one has

$$
\pi_{\omega}\left(\left[\tilde{A}_{t}, \tilde{B}_{t}\right]\right) X \Omega_{\omega}=X \pi_{\omega}\left(\left[\tilde{A}_{t}, \tilde{B}_{t}\right]\right) \Omega_{\omega}
$$

and so for all $\Psi \in \pi_{\omega}(\mathcal{O})^{\prime} \Omega_{\omega}$

$$
\lim _{t \rightarrow \infty} \pi_{\omega}\left(\left[\tilde{A}_{t}, \tilde{B}_{t}\right]\right) \Psi=\left(\int_{-\infty}^{\infty} \omega\left(\left[\tau^{s}(A), B\right]\right) \mathrm{d} s\right) \Psi .
$$

Since $\omega$ is modular $\pi_{\omega}(\mathcal{O})^{\prime} \Omega_{\omega}$ is dense in $\mathcal{H}_{\omega}$ and it follows from the estimate

$$
\sup _{t>0}\left\|\left[\tilde{A}_{t}, \tilde{B}_{t}\right]\right\| \leq \int_{\mathbb{R}}\left\|\left[\tau^{s}(A), B\right]\right\| \mathrm{d} s<\infty,
$$

that (2.31) extends to all $\Psi \in \mathcal{H}_{\omega} . \square$

We are now ready to complete:

Proof of Theorem 1.4. Let $\left\{A_{1}, \cdots, A_{n}\right\} \in \mathfrak{C}_{\text {self }}$. For $j=1, \ldots, n-1$, we set

$$
U_{j t}=\exp \left(-\frac{1}{2}\left[\tilde{A}_{j t}, \tilde{A}_{(j+1) t}+\cdots+\tilde{A}_{n t}\right]\right)
$$

and $U_{t}=U_{1 t} \cdots U_{(n-1) t}$. Clearly, the $U_{j t}$ 's are unitary and repeated use of Proposition 2.1 yields that

$$
\lim _{t \rightarrow \infty}\left\|\mathrm{e}^{\mathrm{i} \tilde{A}_{1 t}} \cdots \mathrm{e}^{\mathrm{i} \tilde{A}_{n t}}-\mathrm{e}^{\mathrm{i}\left(\tilde{A}_{1 t}+\cdots+\tilde{A}_{n t}\right)} U_{t}\right\|=0
$$

and hence,

Proposition 2.3 implies that

$$
\lim _{t \rightarrow \infty}\left|\omega\left(\mathrm{e}^{\mathrm{i} \tilde{A}_{1 t}} \cdots \mathrm{e}^{\mathrm{i} \tilde{A}_{n t}}\right)-\omega\left(\mathrm{e}^{\mathrm{i}\left(\tilde{A}_{1 t}+\cdots+\tilde{A}_{n t}\right)} U_{t}\right)\right|=0
$$

$$
\mathrm{s}-\lim _{t \rightarrow \infty} \pi_{\omega}\left(U_{j t}\right)=\exp \left(-\mathrm{i} \sum_{k=j+1}^{n} \varsigma\left(A_{j}, A_{k}\right)\right)
$$


and so

$$
\mathrm{s}-\lim _{t \rightarrow \infty} \pi_{\omega}\left(U_{t}\right)=\exp \left(-\mathrm{i} \sum_{1 \leq j<k \leq n} \varsigma\left(A_{j}, A_{k}\right)\right) .
$$

Since SQCLT holds, Relations (2.32) and (2.33) yield

$$
\begin{aligned}
\lim _{t \rightarrow \infty} \omega\left(\mathrm{e}^{\left.\mathrm{i} \tilde{A}_{1 t} \cdots \mathrm{e}^{\mathrm{i} \tilde{A}_{n t}}\right)}\right. & =\lim _{t \rightarrow \infty} \omega\left(\mathrm{e}^{\mathrm{i}\left(\tilde{A}_{1 t}+\cdots+\tilde{A}_{n t}\right)} U_{t}\right) \\
& =\lim _{t \rightarrow \infty} \omega\left(\mathrm{e}^{\mathrm{i}\left(\tilde{A}_{1 t}+\cdots+\tilde{A}_{n t}\right)}\right) \exp \left(-\mathrm{i} \sum_{1 \leq j<k \leq n} \varsigma\left(A_{j}, A_{k}\right)\right) \\
& =\exp \left(-\frac{1}{2} L\left(\sum_{k=1}^{n} A_{k}, \sum_{j=1}^{n} A_{j}\right)\right) \exp \left(-\mathrm{i} \sum_{1 \leq j<k \leq n} \varsigma\left(A_{j}, A_{k}\right)\right),
\end{aligned}
$$

and the theorem follows.

\subsection{Norm localization}

For $\epsilon>0$ we denote $D_{\epsilon}=\{z \in \mathbb{C}|| z \mid<\epsilon\}$.

Proposition 2.4 Let $A \in \mathcal{O}_{\text {self }}$ be such that

$$
\int_{-\infty}^{\infty}\left|\omega\left(A \tau^{t}(A)\right)-\omega(A)^{2}\right| \mathrm{d} t<\infty .
$$

Suppose that there exists $\epsilon>0$ such that

$$
\lim _{t \rightarrow \infty} \omega\left(\mathrm{e}^{\mathrm{i} \alpha \tilde{A}_{t}}\right)=\mathrm{e}^{-L(A, A) \alpha^{2} / 2}
$$

for $\alpha \in D_{\epsilon}$. Then (2.34) holds for all $\alpha \in \mathbb{R}$.

Proof. Remark that, for $\alpha=-\mathrm{i} x, \omega\left(\mathrm{e}^{\mathrm{i} \alpha \tilde{A}_{t}}\right)=\omega\left(\mathrm{e}^{x \tilde{A}_{t}}\right)$ and $\mathrm{e}^{-L(A, A) \alpha^{2} / 2}=\mathrm{e}^{L(A, A) x^{2} / 2}$ are moment generating functions. The result then follows from well-known results in classical probability (see the paragraph "Moment generating functions" in Section 30 of [Bil]).

\section{Locally interacting fermions}

In this section we describe the strategy of the proof of our main result, Theorem 1.7, and establish a number of preliminary results needed for the proof. In particular, we shall reduce the proof of Theorem 1.7 to the proof of Theorem 3.5 (stated in Subsection 3.3 and proven in Section 4). Theorem 3.5, which is the main technical result of our paper, concerns only the unperturbed system $\left(\mathcal{O}, \tau_{0}, \omega_{0}\right)$.

\subsection{Strategy}

Suppose that the assumptions of Theorem 1.7 hold and let

$$
A=\sum_{k=1}^{K_{A}} \prod_{j=1}^{n_{k}} a^{*}\left(f_{k j}\right) a\left(g_{k j}\right),
$$


be an element of $\mathfrak{C}$. Clearly,

$$
\omega_{\lambda}^{+}\left(\left(\tilde{A}_{t}\right)^{n}\right)=t^{-n / 2} \int_{[0, t]^{n}} \omega_{\lambda}^{+}\left(\prod_{j=1}^{n}\left(\tau_{\lambda}^{t_{j}}(A)-\omega_{\lambda}^{+}(A)\right)\right) \mathrm{d} t_{1} \cdots \mathrm{d} t_{n} .
$$

The first ingredient of the proof of Theorem 1.7 is:

Theorem 3.1 There exists a finite constant $C_{V, A}$ such that for all $n$,

$$
\sup _{|\lambda| \leq \tilde{\lambda}_{V}, t>0} t^{-n / 2} \int_{[0, t]^{n}}\left|\omega_{\lambda}^{+}\left(\prod_{j=1}^{n}\left(\tau_{\lambda}^{t_{j}}(A)-\omega_{\lambda}^{+}(A)\right)\right)\right| \mathrm{d} t_{1} \cdots \mathrm{d} t_{n} \leq C_{V, A}^{n} n ! .
$$

Remark 1. Our proof also gives an explicit estimate on the constant $C_{V, A}$, see Formula (3.49) below. Remark 2. In the special case $n=2$, Theorem 3.1 yields that for all $t>0$ and $|\lambda| \leq \tilde{\lambda}_{V}$,

$$
\int_{-t}^{t}\left(1-\frac{|s|}{t}\right)\left|\omega_{\lambda}^{+}\left(\left(\tau_{\lambda}^{s}(A)-\omega_{\lambda}^{+}(A)\right)\left(A-\omega_{\lambda}^{+}(A)\right)\right)\right| \mathrm{d} s \leq 2 C_{V, A}^{2} .
$$

As $t \rightarrow \infty$ the monotone convergence theorem yields

$$
\int_{-\infty}^{\infty}\left|\omega_{\lambda}^{+}\left(\left(\tau_{\lambda}^{s}(A)-\omega_{\lambda}^{+}(A)\right)\left(A-\omega_{\lambda}^{+}(A)\right)\right)\right| \mathrm{d} s \leq 2 C_{V, A}^{2} .
$$

In particular, we derive that $\mathfrak{C}$ is CLT-admissible.

The second ingredient of the proof of Theorem 1.7 is:

Theorem 3.2 For $|\lambda| \leq \tilde{\lambda}_{V}$ and all $n \geq 1$,

$$
\lim _{t \rightarrow \infty} \omega_{\lambda}^{+}\left(\left(\tilde{A}_{t}\right)^{n}\right)=\left\{\begin{array}{cc}
\frac{n !}{2^{n / 2}(n / 2) !} L(A, A)^{n / 2} & \text { if } n \text { is even }, \\
0 & \text { if } n \text { is odd } .
\end{array}\right.
$$

Remark. With only notational changes the proof of Theorem 3.2 yields that for all $A_{1}, \cdots, A_{n} \in \mathfrak{C}$,

$$
\lim _{t \rightarrow \infty} \omega_{\lambda}^{+}\left(\tilde{A}_{1 t} \cdots \tilde{A}_{n t}\right)=\omega_{L}\left(\varphi_{L}\left(A_{1}\right) \cdots \varphi_{L}\left(A_{n}\right)\right),
$$

where the r.h.s. is defined by (1.6).

Given Theorems 3.1 and 3.2, we can complete:

Proof of Theorem 1.7. Let $A \in \mathfrak{C}_{\text {self }}$. For $\alpha \in \mathbb{C}$ one has

$$
\omega_{\lambda}^{+}\left(\mathrm{e}^{\mathrm{i} \alpha \tilde{A}_{t}}\right)=\sum_{n \geq 0} \frac{(\mathrm{i} \alpha)^{n}}{n !} \omega_{\lambda}^{+}\left(\left(\tilde{A}_{t}\right)^{n}\right) .
$$

Let $\epsilon=1 /\left(2 C_{V, A}\right)$ and suppose that $|\lambda| \leq \tilde{\lambda}_{V}$. Theorems 3.1 and 3.2 yield that

$$
\sup _{|\alpha|<\epsilon, t>0}\left|\omega_{\lambda}^{+}\left(\mathrm{e}^{\mathrm{i} \alpha \tilde{A}_{t}}\right)\right|<\infty
$$

and that for $|\alpha|<\epsilon$,

$$
\lim _{t \rightarrow \infty} \omega_{\lambda}^{+}\left(\mathrm{e}^{\mathrm{i} \alpha \tilde{A}_{t}}\right)=\mathrm{e}^{-L(A, A) \alpha^{2} / 2} .
$$


Proposition 2.4 yields that (3.37) holds for all $\alpha \in \mathbb{R}$, and so SQCLT holds for $\mathfrak{C}$ w.r.t. $\left(\mathcal{O}, \tau_{\lambda}, \omega_{\lambda}^{+}\right)$. Our standing assumption $\operatorname{Ker}(T)=\operatorname{Ker}(I-T)=\{0\}$ ensures that the state $\omega_{0}$ is modular, and since $\omega_{\lambda}^{+}=\omega_{0} \circ \gamma_{\lambda}^{+}$, the state $\omega_{\lambda}^{+}$is also modular. By Theorem 1.6, if $|\lambda| \leq \lambda_{V}$, then $\mathfrak{C}$ is $L^{1}$-asymptotically Abelian for $\tau_{\lambda}$ and it follows from Theorem 1.4 that the QCLT also holds. $\square$

Notice that in the initial step of the proof we did not use the assumption that $A$ is self-adjoint, and so the following weak form of QCLT holds for any $A \in \mathfrak{C}$ :

Corollary 3.3 For any $A \in \mathfrak{C}$ there exists $\epsilon>0$ such that for $|\lambda| \leq \tilde{\lambda}_{V}$ and $|\alpha|<\epsilon$,

$$
\lim _{t \rightarrow \infty} \omega_{\lambda}^{+}\left(\mathrm{e}^{\mathrm{i} \alpha \tilde{A}_{t}}\right)=\mathrm{e}^{-L(A, A) \alpha^{2} / 2} .
$$

In the rest of this section we shall describe the strategy of the proof of Theorems 3.1 and 3.2.

\subsection{The commutator estimate}

We shall need the following result

Theorem 3.4 Suppose that Assumption (A) holds. Let $V \in \mathfrak{O}(\mathfrak{d})_{\text {self }}$ be a perturbation such that $\bar{n}_{V} \geq 2$ and

$$
\max _{f \in \mathcal{F}(V)}\|f\|=1 \text {. }
$$

Let $A=a^{\#}\left(f_{1}\right) \cdots a^{\#}\left(f_{m}\right)$ be a monomial such that $\mathcal{F}(A)=\left\{f_{1}, \cdots, f_{m}\right\} \subset \mathfrak{d}$, and let

$$
\mathcal{C}_{A}^{(n)}\left(s_{1}, \ldots, s_{n}\right)=\left[\tau_{0}^{s_{n}}(V),\left[\cdots,\left[\tau_{0}^{s_{1}}(V), A\right] \cdots\right]\right] .
$$

Then for all $n \geq 0$ there exist a finite index set $\mathcal{Q}_{n}(A)$, monomials $F_{A, q}^{(n)} \in \mathcal{O}$, and scalar functions $G_{A, q}^{(n)}$ such that

$$
\mathcal{C}_{A}^{(n)}\left(s_{1}, \ldots, s_{n}\right)=\sum_{q \in \mathcal{Q}_{n}(A)} G_{A, q}^{(n)}\left(s_{1}, \ldots, s_{n}\right) F_{A, q}^{(n)}\left(s_{1}, \ldots, s_{n}\right)
$$

Moreover,

1. The order of the monomial $F_{A, q}^{(n)}$ does not exceed $2 n\left(\bar{n}_{V}-1\right)+m$.

2. If $m$ is even then the order of $F_{A, q}^{(n)}$ is also even.

3. The factors of $F_{A, q}^{(n)}$ are from

$$
\left\{a^{\#}\left(\mathrm{e}^{\mathrm{i} s h_{0}} g\right) \mid g \in \mathcal{F}(V), s \in\left\{s_{1}, \ldots, s_{n}\right\}\right\} \cup\left\{a^{\#}(g) \mid g \in \mathcal{F}(A)\right\},
$$

The number of factors from the first set does not exceed $n\left(2 \bar{n}_{V}-1\right)$ while the number of factors from the second set does not exceed $m-1$. In particular, $\left\|F_{A, q}^{(n)}\right\| \leq \max \left(1, \max _{f \in \mathcal{F}(A)}\|f\|^{m-1}\right)$.

4. Let $\lambda_{V}$ be given by (1.15). Then

$$
W_{V, A} \equiv \sum_{n=1}^{\infty}\left|\lambda_{V}\right|^{n} \sum_{q \in \mathcal{Q}_{n}(A)} \int_{-\infty<s_{n} \leq \cdots \leq s_{1} \leq 0}\left|G_{A, q}^{(n)}\left(s_{1}, \ldots, s_{n}\right)\right| \mathrm{d} s_{1} \cdots \mathrm{d} s_{n}<\infty .
$$

The proof of Theorem 3.4 is identical to the proof of Theorem 1.1 in [JOP4]. Parts 1-3 are simple and are stated for reference purposes. The Part 4 is a relatively straightforward consequence of the fundamental Botvich-Guta-Maassen integral estimate [BGM] which also gives an explicit estimate on $W_{V, A}$. A pedagogical exposition of the Botvich-Guta-Maassen estimate can be found in [JP6].

If $A$ is as in Theorem 3.4 then

$$
\gamma_{\lambda}^{+}(A)=\lim _{t \rightarrow \infty} \tau_{0}^{-t} \circ \tau_{\lambda}^{t}(A),
$$


can be expanded in a power series in $\lambda$ which converges for $|\lambda| \leq \lambda_{V}$. Indeed, it follows from the Dyson expansion that

$$
\tau_{0}^{-t} \circ \tau_{\lambda}^{t}(A)=A+\sum_{n=1}^{\infty}(\mathrm{i} \lambda)^{n} \int_{-t \leq s_{n} \leq \cdots \leq s_{1} \leq 0}\left[\tau_{0}^{s_{n}}(V),\left[\cdots,\left[\tau_{0}^{s_{1}}(V), A\right] \cdots\right]\right] \mathrm{d} s_{1} \cdots \mathrm{d} s_{n} .
$$

Hence, for $|\lambda| \leq \lambda_{V}$

$$
\gamma_{\lambda}^{+}(A)=A+\sum_{n=1}^{\infty}(\mathrm{i} \lambda)^{n} \sum_{q \in \mathcal{Q}_{n}(A)} \int_{-\infty<s_{n} \leq \cdots \leq s_{1} \leq 0} G_{A, q}^{(n)}\left(s_{1}, \ldots, s_{n}\right) F_{A, q}^{(n)}\left(s_{1}, \ldots, s_{n}\right) \mathrm{d} s_{1} \cdots \mathrm{d} s_{n},
$$

where the series on the right-hand side is norm convergent by Parts 3 and 4 of Theorem 3.4. This expansion will be used in the proof of Theorems 3.1 and 3.2.

\subsection{Quasi-free correlations}

Let $\mathcal{O}, \tau_{0}$ and $\omega_{0}$ be as in Subsection 1.2. We denote by

$$
\varphi(f)=\frac{1}{\sqrt{2}}\left(a(f)+a^{*}(f)\right)
$$

the Fermi field operator associated to $f \in \mathfrak{h}$. The Fermi field operators satisfy the commutation relation

$$
\varphi(f) \varphi(g)+\varphi(g) \varphi(f)=\operatorname{Re}(f, g) \mathbb{1},
$$

and the CAR algebra $\mathcal{O}$ is generated by $\{\varphi(f) \mid f \in \mathfrak{h}\}$. Clearly,

$$
a(f)=\frac{1}{\sqrt{2}}(\varphi(f)+\mathrm{i} \varphi(\mathrm{i} f)), \quad a^{*}(f)=\frac{1}{\sqrt{2}}(\varphi(f)-\mathrm{i} \varphi(\mathrm{i} f)) .
$$

We recall that $\omega_{0}$, the gauge invariant quasi-free state associated to the density operator $T$, is uniquely specified by

$$
\omega_{0}\left(a^{*}\left(f_{n}\right) \cdots a^{*}\left(f_{1}\right) a\left(g_{1}\right) \cdots a\left(g_{m}\right)\right)=\delta_{n, m} \operatorname{det}\left\{\left(g_{i}, T f_{j}\right)\right\} .
$$

Alternatively, $\omega_{0}$ can be described by its action on the Fermi field operators. Let $\mathcal{P}_{n}$ be the set of all permutations $\pi$ of $\{1, \ldots, 2 n\}$ described in Subsection 1.1 (recall (1.5)). Denote by $\epsilon(\pi)$ the signature of $\pi \in \mathcal{P}_{n}$. $\omega_{0}$ is the unique state on $\mathcal{O}$ such that

and

$$
\omega_{0}\left(\varphi\left(f_{1}\right) \varphi\left(f_{2}\right)\right)=\frac{1}{2}\left(f_{1}, f_{2}\right)-\mathrm{i} \operatorname{Im}\left(f_{1}, T f_{2}\right)
$$

$$
\omega_{0}\left(\varphi\left(f_{1}\right) \cdots \varphi\left(f_{n}\right)\right)=\left\{\begin{array}{cl}
\sum_{\pi \in \mathcal{P}_{n / 2}} \epsilon(\pi) \prod_{j=1}^{n / 2} \omega_{T}\left(\varphi\left(f_{\pi(2 j-1)}\right), \varphi\left(f_{\pi(2 j)}\right)\right) & \text { if } n \text { is even; } \\
0 & \text { if } n \text { is odd. }
\end{array}\right.
$$

For any bounded subset $\mathfrak{f} \subset \mathfrak{h}$ we set

$$
M_{\mathfrak{f}}=\sup _{f \in \mathfrak{f}}\|f\|,
$$

and

$$
C_{\mathfrak{f}}=\max \left(1, \sup _{f, g \in \mathfrak{f}} \frac{2}{\|f\|\|g\|} \int_{-\infty}^{\infty}\left|\omega_{0}\left(\varphi(f) \tau_{0}^{t}(\varphi(g))\right)\right| \mathrm{d} t\right),
$$

and we denote by $\mathcal{M}(\mathfrak{f})$ the set of monomials with factors from $\{\varphi(f) \mid f \in \mathfrak{f}\}$. We further say that $A \in \mathcal{M}(\mathfrak{f})$ is of degree at most $k$ if, for some $f_{1}, \ldots, f_{k} \in \mathfrak{f}$, one can write $A=\varphi\left(f_{1}\right) \cdots \varphi\left(f_{k}\right)$.

Theorem 3.5 Suppose that $C_{\mathfrak{f}}<\infty$. Then for any $A_{1}, \ldots, A_{n} \in \mathcal{M}(\mathfrak{f})$ of degrees at most $k_{1}, \ldots, k_{n}$ the following holds: 
1.

$$
\sup _{t>0} t^{-n / 2} \int_{[0, t]^{n}}\left|\omega_{0}\left(\prod_{i=1}^{n}\left(\tau_{0}^{t_{i}}\left(A_{i}\right)-\omega_{0}\left(A_{i}\right)\right)\right)\right| \mathrm{d} t_{1} \cdots \mathrm{d} t_{n} \leq\left(2^{7 / 2} M_{\mathfrak{f}}\right)^{\sum_{i} k_{i}} C_{\mathfrak{f}}^{n} n ! .
$$

2. If $n$ is odd,

$$
\lim _{t \rightarrow \infty} t^{-n / 2} \int_{[0, t]^{n}} \omega_{0}\left(\prod_{i=1}^{n}\left(\tau_{0}^{t_{i}}\left(A_{i}\right)-\omega_{0}\left(A_{i}\right)\right)\right) \mathrm{d} t_{1} \cdots \mathrm{d} t_{n}=0
$$

3. If $n$ is even,

$$
\lim _{t \rightarrow \infty} t^{-n / 2} \int_{[0, t]^{n}} \omega_{0}\left(\prod_{i=1}^{n}\left(\tau_{0}^{t_{i}}\left(A_{i}\right)-\omega_{0}\left(A_{i}\right)\right)\right) \mathrm{d} t_{1} \cdots \mathrm{d} t_{n}=\sum_{\pi \in \mathcal{P}_{n / 2}} \prod_{j=1}^{n / 2} L_{0}\left(A_{\pi(2 j-1)}, A_{\pi(2 j)}\right),
$$

where

$$
L_{0}\left(A_{i}, A_{j}\right)=\int_{-\infty}^{\infty} \omega_{0}\left(\left(\tau_{0}^{t}\left(A_{i}\right)-\omega_{0}\left(A_{i}\right)\right)\left(A_{j}-\omega_{0}\left(A_{j}\right)\right)\right) \mathrm{d} t
$$

Remark. As in Remark 2 after Theorem 3.1, Part 1 of the previous theorem with $n=2$ implies that

$$
\int_{-\infty}^{\infty}\left|\omega_{0}\left(\left(\tau_{0}^{t}\left(A_{i}\right)-\omega_{0}\left(A_{i}\right)\right)\left(A_{j}-\omega_{0}\left(A_{j}\right)\right)\right)\right| \mathrm{d} t<\infty
$$

and so $L_{0}\left(A_{i}, A_{j}\right)$ is well defined.

Theorem 3.5 is in essence the main technical result of our paper. Its proof is given in Section 4.

We have formulated Theorem 3.5 in terms of field operators since that allows for a combinatorially natural approach to its proof. Using the identities (3.41) one effortlessly gets the following reformulation which is more convenient for our application.

Denote by $\tilde{\mathcal{M}}(\mathfrak{f})$ the set of monomials with factors from $\left\{a^{\#}(f) \mid f \in \mathfrak{f}\right\} . A \in \mathcal{M}(\mathfrak{f})$ is of degree at most $k$ if, for some $f_{1}, \ldots, f_{k} \in \mathfrak{f}$, one can write $A=a^{\#}\left(f_{1}\right) \cdots a^{\#}\left(f_{k}\right)$. Let

$$
D_{\mathfrak{f}}=\max \left(1, \sup _{f, g \in \mathfrak{f} \cup \text { if }} \frac{2}{\|f\|\|g\|} \int_{-\infty}^{\infty}\left|\omega_{0}\left(\varphi(f) \tau_{0}^{t}(\varphi(g))\right)\right| \mathrm{d} t\right),
$$

Corollary 3.6 Suppose that $D_{\mathfrak{f}}<\infty$. Then for any $A_{1}, \ldots, A_{n} \in \tilde{\mathcal{M}}(\mathfrak{f})$ of degrees at most $k_{1}, \ldots, k_{n}$ the following holds:

1.

$$
\sup _{t>0} t^{-n / 2} \int_{[0, t]^{n}}\left|\omega_{0}\left(\prod_{i=1}^{n}\left(\tau_{0}^{t_{i}}\left(A_{i}\right)-\omega_{0}\left(A_{i}\right)\right)\right)\right| \mathrm{d} t_{1} \cdots \mathrm{d} t_{n} \leq\left(2^{4} M_{\mathfrak{f}}\right)^{\sum_{i} k_{i}} D_{\mathfrak{f}}^{n} n ! .
$$

2. If $n$ is odd,

$$
\lim _{t \rightarrow \infty} t^{-n / 2} \int_{[0, t]^{n}} \omega_{0}\left(\prod_{i=1}^{n}\left(\tau_{0}^{t_{i}}\left(A_{i}\right)-\omega_{0}\left(A_{i}\right)\right)\right) \mathrm{d} t_{1} \cdots \mathrm{d} t_{n}=0
$$

3. If $n$ is even,

$$
\lim _{t \rightarrow \infty} t^{-n / 2} \int_{[0, t]^{n}} \omega_{0}\left(\prod_{i=1}^{n}\left(\tau_{0}^{t_{i}}\left(A_{i}\right)-\omega_{0}\left(A_{i}\right)\right)\right) \mathrm{d} t_{1} \cdots \mathrm{d} t_{n}=\sum_{\pi \in \mathcal{P}_{n / 2}} \prod_{j=1}^{n / 2} L_{0}\left(A_{\pi(2 j-1)}, A_{\pi(2 j)}\right),
$$

where $L_{0}\left(A_{i}, A_{k}\right)$ is defined by (3.42). 
Note that if $\mathfrak{c}$ is as in Subsection 1.2 and $\mathfrak{f}$ is a finite subset of $\mathfrak{c}$, then $C_{\mathfrak{f}}<\infty$ and $D_{\mathfrak{f}}<\infty$.

After this paper was completed we have learned of a beautiful paper [De1] which is perhaps deepest among early works on quantum central limit theorems (Dereziński's work was motivated by [Ha1, Ha2, Ru1, HL1, HL2, Da2]). In relation to our work, in [De1] Theorem 3.5 was proven in the special case $k_{1}=\cdots=k_{n}=2$ of quadratic interactions. This suffices for the proof of SQCLT for quasi-free dynamics and for observables which are polynomials in Fermi fields. The proofs of Parts (2) and (3) of Theorem 3.5 are not that much different in the general case $k_{j} \geq 2$. The key difference is in Part (1) which in the quadratic case follows easily from Stirling's formula. To prove Part (1) for any $k_{j} \geq 2$ is much more difficult and the bulk of the proof of Theorem 3.5 in Section 4 is devoted to this estimate. The proof of QCLT for locally interacting fermionic systems critically depends on this result.

\subsection{Proofs of Theorems 3.1 and 3.2}

In this subsection we shall show that Theorems 3.4 and 3.5 imply Theorems 3.1 and 3.2, thereby reducing the proof of Theorem 1.7 to the proof of Theorem 3.5.

If $\eta$ is a state, we shall denote

$$
\eta_{\mathrm{T}}\left(A_{1}, \ldots, A_{n}\right) \equiv \eta\left(\left(A_{1}-\eta\left(A_{1}\right)\right) \ldots\left(A_{n}-\eta\left(A_{n}\right)\right)\right)
$$

Let

$$
A=\sum_{k=1}^{K_{A}} A_{k}, \quad A_{k}=\prod_{j=1}^{n_{k}} a^{*}\left(f_{k j}\right) a\left(g_{k j}\right),
$$

be an element of $\mathfrak{C}$. Without loss of generality we may assume that $\max _{f \in \mathcal{F}(A)}\|f\|=1$. With

$$
\begin{gathered}
\mathfrak{f}=\left\{\mathrm{e}^{\mathrm{i} s h_{0}} f \mid f \in \mathcal{F}(V) \cup \mathcal{F}(A), s \in \mathbb{R}\right\}, \\
D_{V, A}=\max \left(1, \max _{f, g \in \mathcal{F}(V) \cup \mathcal{F}(A)} \frac{1}{\|f\|\|g\|} \int_{-\infty}^{\infty}\left(2^{-1}\left|\left(f, \mathrm{e}^{\mathrm{i} t h_{0}} g\right)\right|+\left|\left(f, \mathrm{e}^{\mathrm{i} t h_{0}} T g\right)\right|\right) \mathrm{d} t\right),
\end{gathered}
$$

we clearly have $M_{\mathfrak{f}}=1$ and $D_{\mathfrak{f}} \leq D_{V, A}$.

Proof of Theorem 3.1. For $|\lambda| \leq \lambda_{V}$,

$$
\omega_{\lambda \mathrm{T}}^{+}\left(\tau_{\lambda}^{t_{1}}(A), \ldots, \tau_{\lambda}^{t_{n}}(A)\right)=\sum_{k_{1}, \ldots, k_{n}=1}^{K_{A}} \omega_{0 \mathrm{~T}}\left(\tau_{0}^{t_{1}} \circ \gamma_{\lambda}^{+}\left(A_{k_{1}}\right), \ldots, \tau_{0}^{t_{n}} \circ \gamma_{\lambda}^{+}\left(A_{k_{n}}\right)\right),
$$

and the expansion (3.40) yields that

$$
\tau_{0}^{t} \circ \gamma_{\lambda}^{+}\left(A_{k}\right)-\omega_{0} \circ \gamma_{\lambda}^{+}\left(A_{k}\right)=\sum_{j \geq 0}(\mathrm{i} \lambda)^{j} \sum_{q \in \mathcal{Q}_{j}\left(A_{k}\right)} \int_{\Delta_{j}} G_{A_{k}, q}^{(j)}(s)\left(\tau_{0}^{t}\left(F_{A_{k}, q}^{(j)}(s)\right)-\omega_{0}\left(F_{A_{k}, q}^{(j)}(s)\right)\right) \mathrm{d} s,
$$

where $\Delta_{j}$ denotes the simplex $\left\{s=\left(s_{1}, \ldots, s_{j}\right) \in \mathbb{R}^{j} \mid-\infty<s_{j}<\cdots<s_{1}<0\right\}$. We have adopted the convention that $Q_{0}\left(A_{k}\right)$ is a singleton, that $G_{A_{k}, q}^{(0)}=1$ and that $F_{A_{k}, q}^{(0)}=A_{k}$. Moreover, integration over the empty simplex $\Delta_{0}$ is interpreted as the identity map. Applying Fubini's theorem we get

$$
\begin{gathered}
t^{-n / 2} \int_{[0, t]^{n}} \omega_{0 \mathrm{~T}}\left(\tau_{0}^{t_{1}} \circ \gamma_{\lambda}^{+}\left(A_{k_{1}}\right), \ldots, \tau_{0}^{t_{n}} \circ \gamma_{\lambda}^{+}\left(A_{k_{n}}\right)\right) \mathrm{d} t_{1} \cdots \mathrm{d} t_{n}=\sum_{j_{1}, \ldots, j_{n} \geq 0}(\mathrm{i} \lambda)^{j_{1}+\cdots+j_{n}} \sum_{q_{1} \in Q_{j_{1}}\left(A_{k_{1}}\right), \ldots, q_{n} \in Q_{j_{n}}\left(A_{k_{n}}\right)} \\
\int_{\Delta_{j_{1}}} \mathrm{~d} s_{1} \cdots \int_{\Delta_{j_{n}}} \mathrm{~d} s_{n}\left(\prod_{l=1}^{n} G_{A_{k_{l}} q_{l}}^{\left(j_{l}\right)}\left(s_{l}\right)\right) C_{t}\left(j, q, s ; A_{k_{1}}, \ldots, A_{k_{n}}\right),
\end{gathered}
$$


where we have set

$$
C_{t}\left(j, q, s ; A_{k_{1}}, \ldots, A_{k_{n}}\right)=t^{-n / 2} \int_{[0,]^{n}} \omega_{0 T}\left(\tau_{0}^{t_{1}}\left(F_{A_{k_{1}} q_{1}}^{\left(j_{1}\right)}\left(s_{1}\right)\right), \ldots, \tau_{0}^{t_{n}}\left(F_{A_{k_{n}} q_{n}}^{\left(j_{n}\right)}\left(s_{n}\right)\right)\right) \mathrm{d} t_{1} \cdots \mathrm{d} t_{n} .
$$

We derive from Corollary 3.6 and Theorem 3.4 that

$$
\left|C_{t}\left(j, q, s ; A_{k_{1}}, \ldots, A_{k_{n}}\right)\right| \leq 2^{8\left(\bar{n}_{V}-1\right) \sum_{l=1}^{n} j_{l}}\left(2^{8 \bar{n}_{A}} D_{\mathfrak{f}}\right)^{n} n !,
$$

holds for $t>0$. Using this bound we further get from (3.46)

$$
\begin{aligned}
& \sup _{t>0} t^{-n / 2} \int_{[0, t]^{n}}\left|\omega_{0 \mathrm{~T}}\left(\tau_{0}^{t_{1}} \circ \gamma_{\lambda}^{+}\left(A_{k_{1}}\right), \ldots, \tau_{0}^{t_{n}} \circ \gamma_{\lambda}^{+}\left(A_{k_{n}}\right)\right)\right| \mathrm{d} t_{1} \cdots \mathrm{d} t_{n} \\
& \leq \prod_{l=1}^{n}\left(2^{8 \bar{n}_{A}} D_{\mathfrak{f}} \sum_{j_{l} \geq 0}\left|2^{8\left(\bar{n}_{V}-1\right)} \lambda\right|^{j_{l}} \sum_{q_{l} \in Q_{j_{l}}\left(A_{k_{l}}\right)_{\Delta_{l}}} \int_{\mathcal{L}_{l}}\left|G_{A_{k_{l}} q_{l}}^{\left(j_{l}\right)}\left(s_{l}\right)\right| \mathrm{d} s_{l}\right) n !
\end{aligned}
$$

For $|\lambda| \leq \tilde{\lambda}_{V}$ we have (recall Definitions (1.17) and (3.39)),

$$
\sum_{j_{l} \geq 0}\left|2^{8\left(\bar{n}_{V}-1\right)} \lambda\right|^{j_{l}} \sum_{q_{l} \in Q_{j_{l}}\left(A_{k_{l}}\right)} \int_{\Delta_{l}}\left|G_{A_{k_{l}} q_{l}}^{\left(j_{l}\right)}\left(s_{l}\right)\right| \mathrm{d} s_{l} \leq 1+W_{V, A_{k_{l}}} .
$$

By Theorem 3.4, the right hand side of this inequality is finite. Combining this bound with (3.44) and (3.48) we finally obtain

$$
\sup _{|\lambda|<\tilde{\lambda}_{V}, t>0} t^{-n / 2} \int_{[0, t]^{n}}\left|\omega_{\lambda \mathrm{T}}^{+}\left(\tau_{\lambda}^{t_{1}}(A), \ldots, \tau_{\lambda}^{t_{n}}(A)\right)\right| \mathrm{d} t_{1} \cdots \mathrm{d} t_{n} \leq\left(2^{8 \bar{n}_{A}} D_{\mathfrak{f}} \sum_{k=1}^{K_{A}}\left(1+W_{V, A_{k}}\right)\right)^{n} n !,
$$

which concludes the proof. $\square$

The above proof gives that in Theorem 3.1 one may take

$$
C_{V, A}=2^{8 \bar{n}_{A}} D_{V, A} \sum_{j=1}^{K_{A}}\left(1+W_{V, A_{k}}\right) .
$$

For an explicit estimate on $W_{V, A_{k}}$ we refer the reader to [JOP4].

Proof of Theorem 3.2. Note that

$$
\omega_{\lambda}^{+}\left(\left(\tilde{A}_{t}\right)^{n}\right)=\sum_{k_{1}, \ldots, k_{n}=1}^{K_{A}} t^{-n / 2} \int_{[0, t]^{n}} \omega_{0 \mathrm{~T}}\left(\tau_{0}^{t_{1}} \circ \gamma_{\lambda}^{+}\left(A_{k_{1}}\right), \ldots, \tau_{0}^{t_{n}} \circ \gamma_{\lambda}^{+}\left(A_{k_{n}}\right)\right) \mathrm{d} t_{1} \cdots \mathrm{d} t_{n} .
$$

In the proof of Theorem 3.1 we have established that the power series (3.46) converges uniformly for $|\lambda| \leq \tilde{\lambda}_{V}$ and $t>0$. Suppose first that $n$ is odd. Corollary 3.6 yields that

$$
\lim _{t \rightarrow \infty} C_{t}\left(j, q, s ; A_{k_{1}}, \ldots, A_{k_{n}}\right)=0
$$

By (3.47) and Part 3 of Theorem 3.4 we can apply the dominated convergence theorem to the $s$-integration in (3.46) to conclude that each term of this power series vanishes as $t \rightarrow \infty$, and so

$$
\lim _{t \rightarrow \infty} \omega_{\lambda}^{+}\left(\left(\tilde{A}_{t}\right)^{n}\right)=0,
$$

for $|\lambda| \leq \tilde{\lambda}_{V}$ 
If $n$ is even, Corollary 3.6 yields

$$
\begin{aligned}
& \lim _{t \rightarrow \infty} C_{t}\left(j, q, s ; A_{k_{1}}, \ldots, A_{k_{n}}\right)=\sum_{\pi \in \mathcal{P}_{n / 2}} \prod_{i=1}^{n / 2} L_{0}\left(F_{A_{k_{\pi(2 i-1)}}^{\left(j_{\pi(2 i-1)}\right)}, q_{\pi(2 i-1)}}\left(s_{\pi(2 i-1)}\right), F_{A_{k_{\pi(2 i)}}, q_{\pi(2 i)}}^{\left(j_{\pi(2 i)}\right)}\left(s_{\pi(2 i)}\right)\right) \\
& =\sum_{\pi \in \mathcal{P}_{n / 2}} \int_{\mathbb{R}^{n / 2}} \prod_{i=1}^{n / 2} \omega_{0 T}\left(\tau_{0}^{t_{i}}\left(F_{A_{k_{\pi(2 i-1)}}, q_{\pi(2 i-1)}}^{\left(j_{\pi(2 i-1)}\right)}\left(s_{\pi(2 i-1)}\right)\right), F_{A_{k_{\pi(2 i)}}, q_{\pi(2 i)}}^{\left(j_{\pi(2 i)}\right)}\left(s_{\pi(2 i)}\right)\right) \mathrm{d} t_{1} \cdots \mathrm{d} t_{n / 2} .
\end{aligned}
$$

The estimate (3.47) (applied in the case $n=2$ ) yields that

$$
\int_{\mathbb{R}}\left|\omega_{0 T}\left(\tau_{0}^{t}\left(F_{A_{k}, q}^{(j)}(s)\right), F_{A_{k^{\prime}}, q^{\prime}}^{\left(j^{\prime}\right)}\left(s^{\prime}\right)\right)\right| \mathrm{d} t \leq\left(2^{8 \bar{n}_{A}+1 / 2} D_{\mathfrak{f}}\right)^{2} 2^{8\left(\bar{n}_{V}-1\right)\left(j+j^{\prime}\right)},
$$

from which we obtain

$$
\left|\lim _{t \rightarrow \infty} C_{t}\left(j, q, s ; A_{k_{1}}, \ldots, A_{k_{n}}\right)\right| \leq\left(2^{8 \bar{n}_{A}+1 / 2} D_{\mathfrak{f}}\right)^{n} 2^{8\left(\bar{n}_{V}-1\right) \sum_{i} j_{i}} .
$$

Arguing as in the previous case we get, for $|\lambda| \leq \tilde{\lambda}_{V}$, the expansion

$$
\begin{aligned}
& \lim _{t \rightarrow \infty} t^{-n / 2} \int_{[0, t]^{n}} \omega_{0 \mathrm{~T}}\left(\tau_{0}^{t_{1}} \circ \gamma_{\lambda}^{+}\left(A_{k_{1}}\right), \ldots, \tau_{0}^{t_{n}} \circ \gamma_{\lambda}^{+}\left(A_{k_{n}}\right)\right) \mathrm{d} t_{1} \cdots \mathrm{d} t_{n / 2} \\
& =\sum_{j_{1}, \ldots, j_{n} \geq 0}(\mathrm{i} \lambda)^{j_{1}+\cdots+j_{n}} \sum_{q_{1} \in \mathcal{Q}_{j_{1}}\left(A_{k_{1}}\right), \cdots, q_{n} \in \mathcal{Q}_{j_{n}}\left(A_{k_{n}}\right)} \int_{\Delta_{j_{1}}} \mathrm{~d} s_{1} \cdots \int_{\Delta_{j_{n}}} \mathrm{~d} s_{n}\left(\prod_{l=1}^{n} G_{A_{k_{l}}, q_{l}}^{\left(j_{l}\right)}\left(s_{l}\right)\right) \\
& \sum_{\pi \in \mathcal{P}_{n / 2}} \int_{\mathbb{R}^{n / 2}} \prod_{i=1}^{n / 2} \omega_{0 T}\left(\tau_{0}^{t_{i}}\left(F_{A_{k_{\pi(2 i-1)}}}^{\left(j_{\pi(2 i-1)}\right)}, q_{\pi(2 i-1)}\left(s_{\pi(2 i-1)}\right)\right), F_{A_{k_{\pi(2 i)}}, q_{\pi(2 i)}}^{\left(j_{\pi(2 i)}\right)}\left(s_{\pi(2 i)}\right)\right) \mathrm{d} t_{1} \cdots \mathrm{d} t_{n / 2} .
\end{aligned}
$$

By Fubini's theorem, this can be rewritten as

$$
\begin{gathered}
\sum_{\pi \in \mathcal{P}_{n / 2}} \int_{\mathbb{R}^{n / 2}}\left[\sum_{j_{1}, \ldots, j_{n} \geq 0}(\mathrm{i} \lambda)^{j_{1}+\cdots+j_{n}} \sum_{q_{1} \in \mathcal{Q}_{j_{1}}\left(A_{k_{1}}\right), \cdots, q_{n} \in \mathcal{Q}_{j_{n}}\left(A_{k_{n}}\right)_{\Delta_{j_{1}}}} \mathrm{~d} s_{1} \cdots \iint_{\Delta_{j_{n}}} \mathrm{~d} s_{n}\right. \\
\left.\left(\prod_{l=1}^{n} G_{A_{k_{l}}, q_{l}}^{\left(j_{l}\right)}\left(s_{l}\right)\right) \prod_{i=1}^{n / 2} \omega_{0 T}\left(\tau_{0}^{t_{i}}\left(F_{A_{k_{\pi(2 i-1)}}^{\left(j_{\pi(2 i-1)}\right)}, q_{\pi(2 i-1)}}\left(s_{\pi(2 i-1)}\right)\right), F_{\left.A_{k_{\pi(2 i)}}, q_{\pi(2 i)}\right)}^{\left(j_{\pi(2 i)}\right)}\left(s_{\pi(2 i)}\right)\right)\right] \mathrm{d} t_{1} \cdots \mathrm{d} t_{n / 2} .
\end{gathered}
$$

By Expansion (3.40), the expression inside the square brackets is

$$
\prod_{i=1}^{n / 2} \omega_{0 T}\left(\tau_{0}^{t_{i}} \circ \gamma_{\lambda}^{+}\left(A_{k_{\pi(2 i-1)}}\right), \gamma_{\lambda}^{+}\left(A_{k_{\pi(2 i)}}\right)\right)=\prod_{i=1}^{n / 2} \omega_{\lambda T}^{+}\left(\tau^{t_{i}}\left(A_{k_{\pi(2 i-1)}}\right), A_{k_{\pi(2 i)}}\right),
$$

so that, by (3.50),

$$
\begin{aligned}
\lim _{t \rightarrow \infty} \omega_{\lambda}^{+}\left(\left(\tilde{A}_{t}\right)^{n}\right) & =\sum_{k_{1}, \ldots, k_{n}=1}^{K_{A}} \sum_{\pi \in \mathcal{P}_{n / 2}} \prod_{i=1}^{n / 2}\left(\int_{\mathbb{R}} \omega_{\lambda T}^{+}\left(\tau^{t}\left(A_{k_{\pi(2 i-1)}}\right), A_{k_{\pi(2 i)}}\right) \mathrm{d} t\right) \\
& =\sum_{\pi \in \mathcal{P}_{n / 2}} \prod_{i=1}^{n / 2}\left(\int_{\mathbb{R}} \omega_{\lambda T}^{+}\left(\tau^{t}(A), A\right) \mathrm{d} t\right) \\
& =\frac{n !}{2^{n / 2}(n / 2) !} L(A, A)^{n / 2} .
\end{aligned}
$$




\section{Proof of Theorem 3.5}

For notational simplicity throughout this section we shall drop the subscript 0 and write $h$ for $h_{0}, \tau$ for $\tau_{0}, \omega$ for $\omega_{0}$. We shall also use the shorthand (3.43).

\subsection{Graphs, pairings and Pfaffians}

An graph is a pair of sets $g=(V, E)$ where $E$ is a set of 2-elements subsets of $V$. The elements of $V$ are called points or vertices of $g$, those of $E$ are its edges. Abusing notation, we shall write $v \in g$ for vertices of $g$ and $e \in g$ for its edges. If $v \in e \in g$ we say that the edge $e$ is incident to the vertex $v$. If the edge $e$ is incident to the vertices $u$ and $v$ we write $e=u v$ and say that the edge $e$ connects $u$ to $v$. The degree of a vertex $v \in g$ is the number of distinct edges $e \in g$ incident to $v$. A graph is $k$-regular if all its vertices share the same degree $k$. A vertex $v \in g$ of degree 0 is said to be isolated. A path on $g$ is a sequence $\left(v_{0}, e_{1}, v_{1}, e_{2}, \ldots, e_{n}, v_{n}\right)$ where $v_{i} \in V, e_{i} \in E$ and $e_{i}=v_{i-1} v_{i}$. We say that such a path connects the vertices $v_{0}$ and $v_{n}$. If $v_{0}=v_{n}$ the path is closed and is called a loop. The graph $g$ is connected if, given any pair $v, v^{\prime} \in V$ there is a path on $g$ which connects $v$ and $v^{\prime}$. A connected graph without loops is a tree.

A graph $g^{\prime}=\left(V^{\prime}, E^{\prime}\right)$ is a subgraph of the graph $g=(V, E)$ if $V^{\prime} \subset V$ and $E^{\prime} \subset E$. A subgraph $g^{\prime}$ of $g$ is said to be spanning $g$ if $V^{\prime}=V$. A connected graph $g$ has a spanning tree i.e., a subgraph which is spanning and is a tree.

Let $g=(V, E)$ be a graph. To a subset $W \subset V$ we associate a subgraph $g_{\mid W}=\left(W, E_{\mid W}\right)$ of $g$ by setting $E_{\mid W}=\{e=u v \in$ $E \mid u, v \in W\}$. Given two graphs $g_{1}=\left(V_{1}, E_{1}\right)$ and $g_{2}=\left(V_{2}, E_{2}\right)$ such that $V_{1}$ and $V_{2}$ are disjoint we denote by $g_{1} \vee g_{2}$ the joint graph $\left(V_{1} \cup V_{2}, E_{1} \cup E_{2}\right)$.

Let $g=(V, E)$ be a graph and $\Pi=\left\{V_{1}, \ldots, V_{n}\right\}$ a partition of $V$. The set

$$
E / \Pi=\left\{V_{i} V_{j} \mid \text { there are } u \in V_{i}, v \in V_{j} \text { such that } u v \in E\right\} .
$$

defines a graph $g / \Pi=(\Pi, E / \Pi)$. We say that $g / \Pi$ is the $\Pi$-skeleton of $g$.

A graph $g=(V, E)$ is said to be $\left(V_{1}, V_{2}\right)$-bipartite if there is a partition $V=V_{1} \cup V_{2}$ such that all edges $e \in E$ connect a vertex of $V_{1}$ to a vertex of $V_{2}$.

A pairing on a set $V$ is a graph $p=(V, E)$ such that every vertex $v \in V$ belongs to exactly one edge $e \in E$. Equivalently, $p=(V, E)$ is a pairing if $E$ is a partition of $V$ or if it is 1-regular. We denote by $\mathcal{P}(V)$ the set of all pairings on $V$. Clearly, only sets $V$ of even parity $|V|=2 n$ admit pairings and in this case one has

$$
|\mathcal{P}(V)|=\frac{(2 n) !}{2^{n} n !}=(2 n-1) ! !
$$

If the set $V=\left\{v_{1}, \ldots, v_{2 n}\right\}$ is completely ordered, $v_{1}<v_{2}<\cdots<v_{2 n}$, writing

$$
E=\left\{\pi\left(v_{1}\right) \pi\left(v_{2}\right), \pi\left(v_{3}\right) \pi\left(v_{4}\right), \ldots, \pi\left(v_{2 n-1}\right) \pi\left(v_{2 n}\right)\right\},
$$

sets a one-to-one correspondence between pairings $p=(V, E)$ and permutations $\pi \in S_{V}$ such that $\pi\left(v_{2 i-1}\right)<\pi\left(v_{2 i}\right)$ and $\pi\left(v_{2 i-1}\right)<\pi\left(v_{2 i+1}\right)$ for $i=1, \ldots, n$ (compare with (1.5)). In the sequel we will identify the two pictures and denote by $p$ the permutation of $V$ associated to the pairing $p$. In particular, the signature $\varepsilon(p)$ of a pairing $p$ is given by the signature of the corresponding permutation. A diagrammatic representation of a pairing $p \in \mathcal{P}(V)$ is obtained by drawing the vertices $v_{1}, \ldots, v_{2 n}$ as $2 n$ consecutive points on a line. Each edge $e \in p$ is drawn as an arc connecting the corresponding points above this line (see Figure 1). It is well known that the signature of $p$ is then given by $\varepsilon(p)=(-1)^{k}$ where $k$ is the total number of intersection points of these arcs.

If $V=V_{1} \cup V_{2}$ is a partition of $V$ into two equipotent subsets we denote by $\mathcal{P}\left(V_{1}, V_{2}\right) \subset \mathcal{P}(V)$ the corresponding set of $\left(V_{1}, V_{2}\right)$-bipartite pairings and note that

$$
\left|\mathcal{P}\left(V_{1}, V_{2}\right)\right|=n !
$$

If $V_{1}=\left\{v_{1}, \ldots, v_{n}\right\}$ and $V_{2}=\left\{v_{n+1}, \ldots, v_{2 n}\right\}$ are completely ordered by $v_{1}<\cdots<v_{n}<\cdots<v_{2 n}$ then $p\left(v_{2 i-1}\right)=v_{i}$ and $\sigma\left(v_{n+i}\right)=p\left(v_{2 i}\right)$ for $1 \leq i \leq n$ defines a one-to-one correspondence between bipartite pairings $p \in \mathcal{P}\left(V_{1}, V_{2}\right)$ and permutations $\sigma \in S_{V_{2}}$. A simple calculation shows that $\varepsilon(p)=(-1)^{n(n-1) / 2} \varepsilon(\sigma)$. 


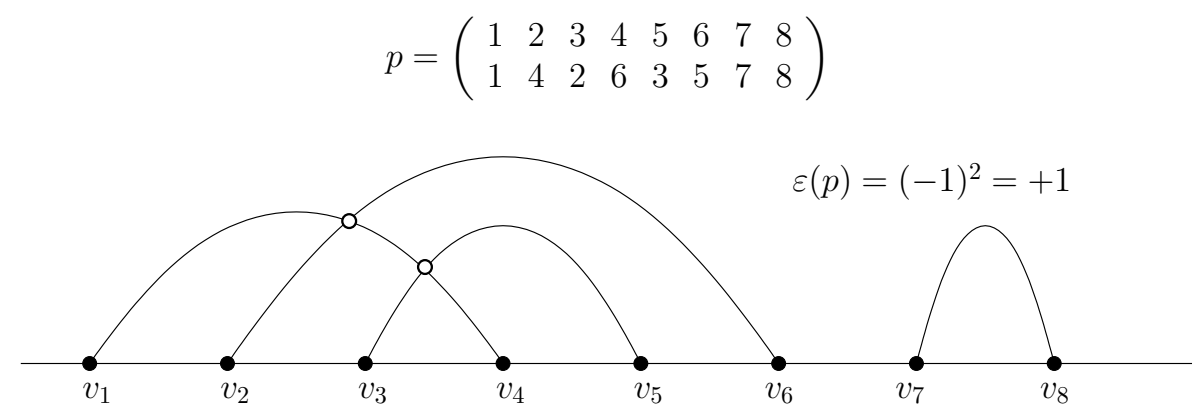

Figure 1: Diagrammatic representation of a pairing $p$

In the special case $V=\{1, \ldots, 2 n\}, V_{1}=\{1, \ldots, n\}$ and $V_{2}=\{n+1, \ldots, 2 n\}$ we shall set $\mathcal{P}(V)=\mathcal{P}_{n}$ and $\mathcal{P}\left(V_{1}, V_{2}\right)=$ $\widetilde{\mathcal{P}}_{n}$.

The Pfaffian of a $2 n \times 2 n$ skew-symmetric matrix $M$ is defined by

$$
\operatorname{Pf}(M)=\sum_{p \in \mathcal{P}_{n}} \varepsilon(p) \prod_{i=1}^{n} M_{p(2 i-1) p(2 i)} .
$$

If $B$ is a $n \times n$ matrix and

$$
M=\left[\begin{array}{cc}
0 & B \\
-B^{T} & 0
\end{array}\right]
$$

then only bipartite pairings $p \in \widetilde{\mathcal{P}}_{n}$ contribute to the Pfaffian of $M$ which reduces to

$$
\begin{aligned}
\operatorname{Pf}(M) & =\sum_{p \in \widetilde{\mathcal{P}}_{n}} \varepsilon(p) \prod_{i=1}^{n} B_{p(2 i-1) p(2 i)} \\
& =\sum_{\sigma \in S_{n}}(-1)^{n(n-1) / 2} \varepsilon(\sigma) \prod_{i=1}^{n} B_{i \sigma(i)} \\
& =(-1)^{n(n-1) / 2} \operatorname{det}(B) .
\end{aligned}
$$

\subsection{Truncating quasi-free expectations}

Let $V \subset \mathfrak{h}$ be finite and totally ordered. To any subset $W \subset V$ we assign the monomial

$$
\Phi(W) \equiv \prod_{u \in W} \varphi(u),
$$

where the product is ordered from left to right in increasing order of the index $u$.

Let $\omega$ be a gauge invariant quasi-free state on $\operatorname{CAR}(\mathfrak{h})$. We define a $|V| \times|V|$ skew-symmetric matrix $\Omega$ by setting

$$
\Omega_{u v} \equiv \omega(\varphi(u) \varphi(v))
$$

for $u, v \in V$ and $u<v$. We also denote by $\Omega^{W}$ the sub-matrix of $\Omega$ whose row and column indices belong to $W$. Then we have

$$
\omega(\Phi(W))= \begin{cases}\operatorname{Pf}\left(\Omega^{W}\right) & \text { if }|W| \text { is even } \\ 0 & \text { otherwise. }\end{cases}
$$

If $|W|$ is even, assigning to any pairing $p \in \mathcal{P}(W)$ the weight

$$
\Omega(p) \equiv \prod_{\substack{u v \in p \\ u<v}} \Omega_{u v},
$$




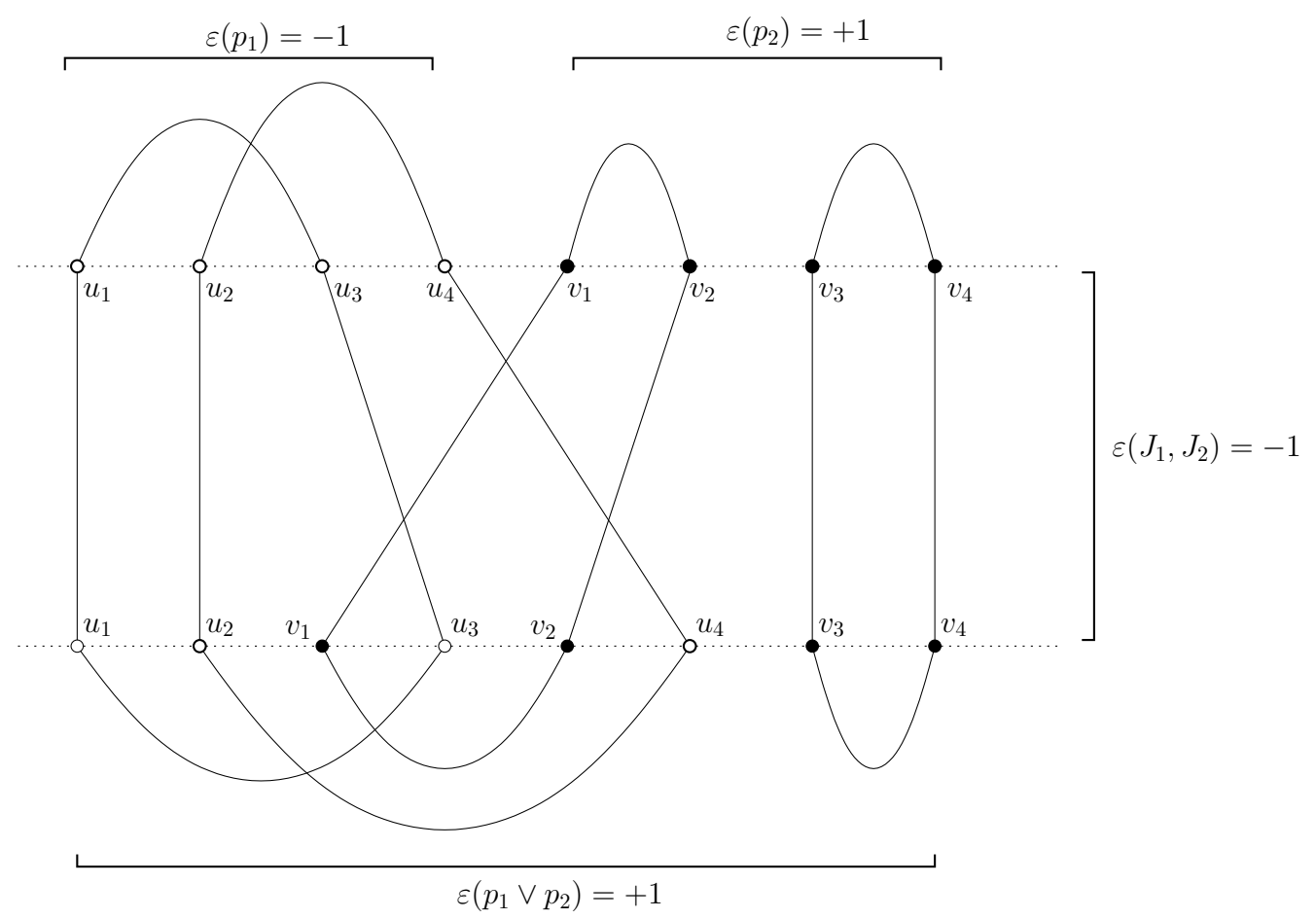

Figure 2: Proof of Lemma 4.1

we can rewrite Equ. (4.54) as

$$
\omega(\Phi(W))=\sum_{p \in \mathcal{P}(W)} \varepsilon(p) \Omega(p) .
$$

The following simple lemma is our fundamental tool when dealing with such expansions.

Lemma 4.1 Let $W_{1}=\left\{u_{1}, \ldots, u_{r}\right\}$ and $W_{2}=\left\{v_{1}, \ldots, v_{s}\right\}$ be disjoint even subsets of $V$ such that $u_{1}<u_{2}<\cdots<u_{r}$ and $v_{1}<v_{2}<\cdots<v_{s}$. Denote by $\varepsilon\left(W_{1}, W_{2}\right)$ the signature of the permutation of $W_{1} \cup W_{2}$ which "orders" the sequence $W_{1} W_{2}$ i.e., which maps the sequence $u_{1}, u_{2}, \cdots u_{r}, v_{1}, v_{2}, \cdots, v_{s}$ into the ordered sequence of elements of $W_{1} \cup W_{2}$. Then, for any $p_{1} \in \mathcal{P}\left(W_{1}\right)$ and $p_{2} \in \mathcal{P}\left(W_{2}\right)$ one has

$$
\Omega\left(p_{1} \vee p_{2}\right)=\Omega\left(p_{1}\right) \Omega\left(p_{2}\right), \quad \varepsilon\left(p_{1} \vee p_{2}\right)=\varepsilon\left(W_{1}, W_{2}\right) \varepsilon\left(p_{1}\right) \varepsilon\left(p_{2}\right) .
$$

Proof. The statement about $\Omega\left(p_{1} \vee p_{2}\right)$ is obvious. To prove the statement about signatures we draw the following diagram (see Figure 2). Draw two parallel lines and on the top one the two diagrams corresponding to the pairings $p_{1}$ and $p_{2}$, one next to the other. On the bottom line draw the diagram representing the pairing $p_{1} \vee p_{2}$ but with the edges drawn below the baseline. Now draw segments connecting each point of the top line with its representant on the bottom line. These segments represent the permutation referred to in the Lemma. Thus, if there are $q$ intersection points of these segments then $\varepsilon\left(W_{1}, W_{2}\right)=(-1)^{q}$. Denote by $j$ the number of intersection points in our diagram lying above the top line and by $j^{\prime}$ the number of those intersections points lying below the bottom line. Then, we have $\varepsilon\left(p_{1}\right) \varepsilon\left(p_{2}\right)=(-1)^{j}$ and $\varepsilon\left(p_{1} \vee p_{2}\right)=(-1)^{j^{\prime}}$. Now observe that our diagram is a disjoint union of closed loops. Thus, it has an even number of intersection points i.e.,

$$
(-1)^{j+q+j^{\prime}}=1
$$

from which the result follows. $\square$ 
Iterating Equ. (4.56) we obtain, for disjoint even subsets $W_{1}, \ldots, W_{k} \subset V$ and arbitrary pairings $p_{i} \in \mathcal{P}\left(W_{i}\right)$, the formulas

$$
\Omega\left(\bigvee_{i=1}^{k} p_{i}\right)=\prod_{i=1}^{k} \Omega\left(p_{i}\right), \quad \varepsilon\left(\bigvee_{i=1}^{k} p_{i}\right)=\varepsilon\left(W_{1}, \ldots, W_{k}\right) \prod_{i=1}^{k} \varepsilon\left(p_{i}\right),
$$

where $\varepsilon\left(W_{1}, \ldots, W_{k}\right)$ denotes the signature of the permutation which "orders" the sequence $W_{1}, \ldots, W_{k}$. Moreover, the recurrence relation

$$
\varepsilon\left(W_{1}, \ldots, W_{k}\right)=\varepsilon\left(W_{1} \cup \cdots \cup W_{k-1}, W_{k}\right) \varepsilon\left(W_{1}, \ldots, W_{k-1}\right)
$$

holds.

If $X, Y$ are subsets of $V$ we write $X<Y$ whenever $\max (X)<\min (Y)$.

Remark. If $W_{1}<W_{2}<\cdots<W_{k}$ it immediately follows from the fact that the $W_{i}$ are even that $\varepsilon\left(W_{\pi(1)}, \ldots, W_{\pi(k)}\right)=1$ for any permutation $\pi \in S_{k}$.

Lemma 4.2 Let $\Pi=\left(V_{1}, \ldots, V_{n}\right)$ be an ordered partition of $V$ by even subsets i.e.,

$$
V_{1}<V_{2}<\cdots<V_{n}, \quad\left|V_{i}\right| \text { even. }
$$

and set $A_{i}=\Phi\left(V_{i}\right)$. Then one has

$$
\omega_{T}\left(A_{1}, \ldots, A_{n}\right)=\sum_{p \in \mathcal{P}(\Pi)} \varepsilon(p) \Omega(p),
$$

where $\mathcal{P}(\Pi)$ denotes the set of pairings $p \in \mathcal{P}(V)$ which have a $\Pi$-skeleton $p / \Pi$ without isolated vertex.

Proof. Expanding the left hand side of Equ. (4.57) we get

$$
\sum_{K \subset I}(-1)^{|K|} \omega\left(\Phi\left(\cup_{i \in I \backslash K} V_{i}\right)\right) \prod_{i \in K} \omega\left(\Phi\left(V_{i}\right)\right),
$$

where $I=\{1, \ldots, n\}$. Using Lemma 4.1 and the remarks following it, we can rewrite this expression as

$$
\sum_{K \subset I}(-1)^{|K|} \sum_{\xi \in \Xi_{K}} \varepsilon(p(\xi)) \Omega(p(\xi))
$$

where we sum over the sets

$$
\Xi_{K} \equiv \mathcal{P}\left(\cup_{i \in I \backslash K} V_{i}\right) \times\left(\prod_{i \in K} \mathcal{P}\left(V_{i}\right)\right),
$$

and, for $\xi=\left(q,\left(p_{i}\right)_{i \in K}\right) \in \Xi_{K}$ we have set $p(\xi)=q \vee\left(\vee_{i \in K} p_{i}\right)$. Let us define

$$
\operatorname{Is}(p) \equiv\left\{i \in I \mid V_{i} \text { is an isolated vertex of } p / \Pi\right\} .
$$

Clearly, if $K \subset I$ and $\xi \in \Xi_{K}$ then $p(\xi) \in \mathcal{P}(V)$ and $K \subset \operatorname{Is}(p(\xi))$. Reciprocally, suppose that $p \in \mathcal{P}(V)$ and $K \subset \operatorname{Is}(p)$. Then the restricted graphs $q=p_{\mid W}$ with $W=\cup_{i \in I \backslash K} V_{i}$ and $p_{i}=p_{\mid V_{i}}$ for $i \in K$ satisfy $\xi=\left(q,\left(p_{i}\right)_{i \in K}\right) \in \Xi_{K}$ and $p(\xi)=p$. We conclude that

$$
\left\{(K, p(\xi)) \mid K \subset I, \xi \in \Xi_{K}\right\}=\{(K, p) \mid p \in \mathcal{P}(V), K \subset \operatorname{Is}(p)\},
$$

and since the map $\xi \mapsto p(\xi)$ is clearly injective we can rewrite the sum (4.58) as

$$
\sum_{p \in \mathcal{P}(V)} \varepsilon(p) \Omega(p) \sum_{K \subset \operatorname{Is}(p)}(-1)^{|K|}
$$

The result follows from the fact that the second sum in the last expression vanishes unless Is $(p)$ is empty. $\square$ 


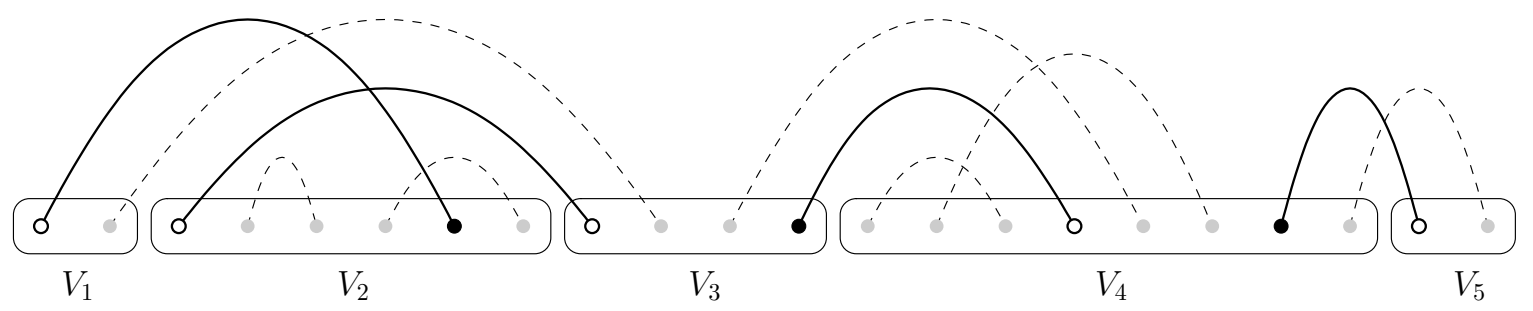

Figure 3: The exit graph $\operatorname{ex}(p)$ (solid lines) for $p \in \mathcal{P}(\Pi)$

\subsection{Resummation}

The setup in this subsection is the same as in the previous one. We consider a fixed ordered partition $\Pi=\left(V_{1}, \ldots, V_{n}\right)$ of $V$ by even subsets as in Lemma 4.2 and fix our attention on the expansion (4.57) of the truncated correlation.

Consider a fixed term in this expansion i.e., a pairing $p \in \mathcal{P}(\Pi)$. Since its skeleton $p / \Pi$ has no isolated point, for each $i \in I=\{1, \ldots, n\}$ the set of edges of $p$ which connect a vertex in $V_{i}$ to a vertex outside $V_{i}$ is not empty. We call exit edge of $p$ from $V_{i}$ the element of this set which contains the smallest vertex in $V_{i}$. The set of all exit edges of $p$ defines a subgraph of $p$ which we denote by $\operatorname{ex}(p)$ (see Figure 3). We also denote by $\operatorname{Ex}(\Pi)=\{\operatorname{ex}(p) \mid p \in \mathcal{P}(\Pi)\}$ the set of all exit graphs. We can rewrite expansion (4.57) as

$$
\omega_{T}\left(A_{1}, \ldots, A_{n}\right)=\sum_{g \in \operatorname{Ex}(\Pi)} \sum_{p \in \mathrm{ex}^{-1}(\{g\})} \varepsilon(p) \Omega(p) .
$$

A given exit graph $g$ can be seen as a pairing on $X(g)=\{u \in V \mid u v \in g$ for some $v \in V\}$. Setting $V(g)=V \backslash X(g)$ and applying Lemma 4.1 we get

$$
\omega_{T}\left(A_{1}, \ldots, A_{n}\right)=\sum_{g \in \operatorname{Ex}(\Pi)} \Omega(g) S(g)
$$

where

$$
S(g) \equiv \sum_{p \in \mathrm{ex}^{-1}(\{g\})} \varepsilon(p) \Omega\left(p_{\mid V(g)}\right) .
$$

Our next result is a partial resummation formula for $S(g)$.

Define the exit point from $V_{i}$ by $x_{i}(g) \equiv \min \left(X(g) \cap V_{i}\right)$. We say that

$$
\theta=\left(X, L, M, M^{\prime}, R\right),
$$

is a $g$-admissible partition of $V$ if $X, L, M, M^{\prime}$ and $R$ are disjoint subsets of $V$ such that

$$
X=X(g), \quad V=X \cup L \cup M \cup M^{\prime} \cup R,
$$

and which, for all $i \in I$, satisfy the following conditions

(1) $\max \left((L \cup M) \cap V_{i}\right)<x_{i}(g)$;

(2) $\min \left(\left(R \cup M^{\prime}\right) \cap V_{i}\right)>x_{i}(g)$;

(3) $\left|L \cap V_{i}\right|$ is even;

(4) $\left|M \cap V_{i}\right|=\left|M^{\prime} \cap V_{i}\right|$.

If $X, Y$ are two subsets of $V$ denote by $\Omega^{X, Y}$ the sub-matrix of $\Omega$ with row (resp. column) indices in $X$ (resp. $Y$ ).

Lemma 4.3 For $g \in \operatorname{Ex}(\Pi)$ one has

$$
S(g)=\sum_{\theta=\left(X, L, M, M^{\prime}, R\right) \in \Theta(g)} \varepsilon(\theta) \omega(\Phi(R)) \prod_{i \in I}\left(\omega\left(\Phi\left(L \cap V_{i}\right)\right) \operatorname{det}\left(\Omega^{M \cap V_{i}, M^{\prime} \cap V_{i}}\right)\right),
$$




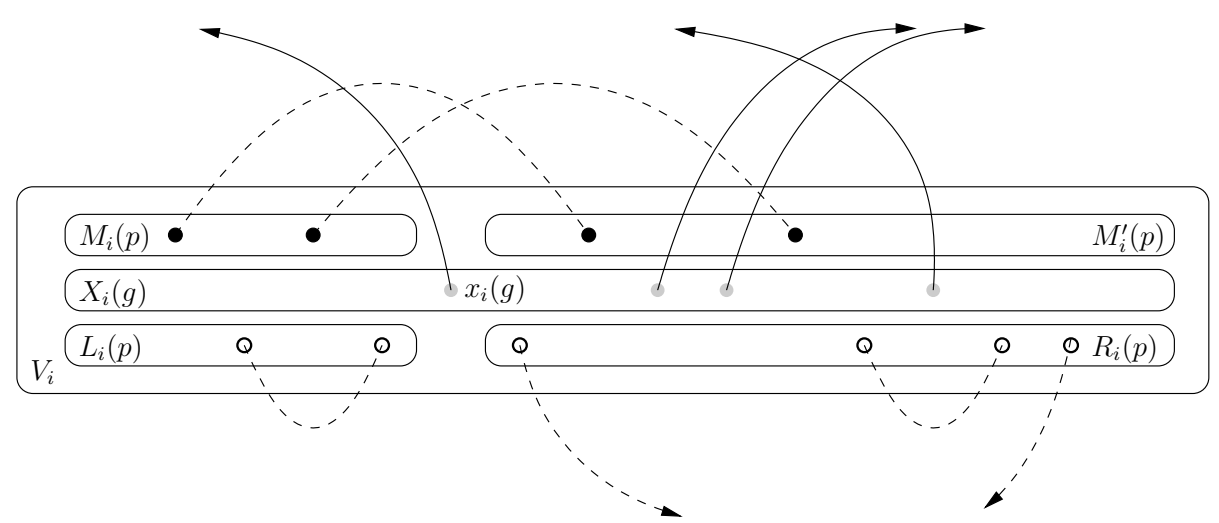

Figure 4: The partition of $V_{i}$ induced by a pairing $p$. Solid lines belong to the exit graph $\operatorname{ex}(p)$.

where $\Theta(g)$ denotes the set of $g$-admissible partitions of $V$ and

$$
\varepsilon(\theta) \equiv \varepsilon\left(X, L \cap V_{1}, \ldots, L \cap V_{n},\left(M \cup M^{\prime}\right) \cap V_{1}, \ldots,\left(M \cup M^{\prime}\right) \cap V_{n}, R\right) \varepsilon\left(g_{\mid X}\right) \prod(-1)^{\left|M \cap V_{i}\right|\left(\left|M \cap V_{i}\right|-1\right) / 2} .
$$

Proof. Let us have a closer look at a pairing $p$ whose exit graph is $g$. What happens in $X_{i}^{i}\left(\xi^{J}\right) \equiv V_{i} \cap X(g)$ is completely determined by $g$. However, the structure of $p_{\mid V_{i}(g)}$ where $V_{i}(g) \equiv V_{i} \cap V(g)$ depends on finer details of $p$. Edges of $p$ which are incident to a vertex in $V_{i}(g)$ located to the left of the exit point $x_{i}(g)$ must connect this vertex to another vertex in $V_{i}(g)$. These edges split in two categories: the ones which connect two vertices on the left of the exit point and the ones which connect a vertex on the left to a vertex on the right. We denote by $L_{i}(p)$ the set of vertices which belong to an edge of the first category, and by $M_{i}(p)$ the vertices located to the left of $x_{i}(g)$ and belonging to an edge of the second one. By $M_{i}^{\prime}(p)$ we denote the set of vertices which are connected to elements of $M_{i}(p)$. This subset of $V_{i}(g)$ is located on the right of the exit point. We group the remaining vertices of $V_{i}(g)$, which are all on the right of the exit point, into a fourth set $R_{i}(p)$. Elements of this set connect among themselves or with elements of $R_{j}(p)$ for some $j \neq i$ (see Figure 4). Setting

$$
L(p) \equiv \bigcup_{i \in I} L_{i}(p), \quad M(p) \equiv \bigcup_{i \in I} M_{i}(p), \quad M^{\prime}(p) \equiv \bigcup_{i \in I} M_{i}^{\prime}(p), \quad R(p) \equiv \bigcup_{i \in I} R_{i}(p),
$$

we obtain a partition

$$
\theta(p) \equiv\left(X(g), L(p), M(p), M^{\prime}(p), R(p)\right),
$$

of $V$ which is clearly $g$-admissible. Moreover, setting

$$
\begin{aligned}
l_{i}(p) \equiv p_{\mid L(p) \cap V_{i}} & \in \mathcal{P}\left(L(p) \cap V_{i}\right), \\
m_{i}(p) \equiv p_{\mid\left(M(p) \cup M^{\prime}(p)\right) \cap V_{i}} & \in \mathcal{P}\left(M(p) \cap V_{i}, M^{\prime}(p) \cap V_{i}\right), \\
r(p) \equiv p_{\mid R(p)} & \in \mathcal{P}(R(p)) .
\end{aligned}
$$

we obtain a map $\Psi$ from $\operatorname{ex}^{-1}(\{g\})$ to the set

$$
\bigcup_{\theta=\left(X, L, M, M^{\prime}, R\right) \in \Theta(g)}\left[\{\theta\} \times\left(\prod_{i \in I} \mathcal{P}\left(L \cap V_{i}\right)\right) \times\left(\prod_{i \in I} \mathcal{P}\left(M \cap V_{i}, M^{\prime} \cap V_{i}\right)\right) \times \mathcal{P}(R)\right] .
$$

Since

$$
p=g \vee\left(\bigvee_{i \in I} l_{i}(p)\right) \vee\left(\bigvee_{i \in I} m_{i}(p)\right) \vee r(p),
$$

$\Psi$ is injective. For any $g$-admissible partition $\theta=\left(X, L, M, M^{\prime}, R\right)$ and any

$$
l_{i} \in \mathcal{P}\left(L \cap V_{i}\right), \quad m_{i} \in \mathcal{P}\left(M \cap V_{i}, M^{\prime} \cap V_{i}\right), \quad r \in \mathcal{P}(R)
$$


the pairing

$$
p=g \vee\left(\bigvee_{i \in I} l_{i}\right) \vee\left(\bigvee_{i \in I} m_{i}\right) \vee r
$$

satisfies

$$
\operatorname{ex}(p)=g, \quad \theta(p)=\theta, \quad l_{i}(p)=l_{i}, \quad m_{i}(p)=m_{i}, \quad r(p)=r .
$$

We conclude that $\Psi$ is bijective. Thus, using Lemma 4.1, we can rewrite the sum $S(g)$ as

$$
\begin{array}{r}
\sum_{\theta=\left(X, L, M, M^{\prime}, R\right) \in \Theta(g)} \varepsilon\left(g_{\mid X}\right) \varepsilon\left(X, L \cap V_{1}, \ldots, L \cap V_{n},\left(M \cup M^{\prime}\right) \cap V_{1}, \ldots,\left(M \cup M^{\prime}\right) \cap V_{n}, R\right) \\
\prod_{i \in I}\left(\sum_{l_{i} \in \mathcal{P}\left(L \cap V_{i}\right)} \varepsilon\left(l_{i}\right) \Omega\left(l_{i}\right)\right) \prod_{i \in I}\left(\sum_{m_{i} \in \mathcal{P}\left(M \cap V_{i}, M^{\prime} \cap V_{i}\right)} \varepsilon\left(m_{i}\right) \Omega\left(m_{i}\right)\right) \sum_{r \in \mathcal{P}(R)} \varepsilon(r) \Omega(r) .
\end{array}
$$

The result now follows from Equ. (4.53) and (4.55).

\subsection{Estimating truncated expectations}

Apart from the entropic factor $|\Theta(g)|$, the following Lemma controls the partial sum $S(g)$.

Lemma 4.4 For $g \in \operatorname{Ex}(\Pi)$ one has

$$
|S(g)| \leq 2^{-|V(g)| / 2}|\Theta(g)| \prod_{v \in V(g)}\|v\| .
$$

Proof. Since

$$
\varphi(f)^{2}=\frac{1}{2}\left\{a^{*}(f), a(f)\right\}=\frac{1}{2}\|f\|^{2},
$$

we have, for any $X \subset V$, the simple bound

$$
|\omega(\Phi(X))| \leq 2^{-|X| / 2} \prod_{v \in X}\|v\| .
$$

Combining this estimate with the following Lemma, the result is an immediate consequence of Formula (4.61). $\square$

Lemma 4.5 Let $B$ be the $k \times k$ matrix defined by $B_{i j}=\omega\left(\varphi\left(u_{i}\right) \varphi\left(v_{j}\right)\right)$. Then, the estimate

$$
|\operatorname{det}(B)| \leq 2^{-k} \prod_{i=1}^{k}\left(\left\|u_{i}\right\|\left\|v_{i}\right\|\right),
$$

holds.

Proof. Let $\div$ be a complex conjugation on $\mathfrak{h}$. The real-linear map

$$
\begin{aligned}
Q: \quad \mathfrak{h} & \rightarrow \mathfrak{h} \oplus \mathfrak{h} \\
f & \mapsto(1-T)^{1 / 2} f \oplus \bar{T}^{1 / 2} \bar{f},
\end{aligned}
$$

is isometric and such that

$$
\omega\left(\varphi\left(u_{i}\right) \varphi\left(v_{j}\right)\right)=\frac{1}{2}\left(\left(u_{i}, v_{j}\right)-\left(u_{i}, T v_{j}\right)+\overline{\left(u_{i}, T v_{j}\right)}\right)=\frac{1}{2}\left(Q u_{i}, Q v_{j}\right) .
$$

It immediately follows that

$$
\operatorname{det}(B)=2^{-k} \omega_{\mathrm{Fock}}\left(a\left(Q u_{1}\right) \cdots a\left(Q u_{k}\right) a^{*}\left(Q v_{k}\right) \cdots a^{*}\left(Q v_{1}\right)\right),
$$


where $\omega_{\text {Fock }}$ denotes the Fock-vacuum state on $\operatorname{CAR}(\mathfrak{h} \oplus \mathfrak{h})$. The fact that

$$
\|a(Q u)\|=\left\|a^{*}(Q u)\right\|=\|Q u\|=\|u\|,
$$

for any $u \in \mathfrak{h}$ yields the result. $\square$

For $u, v \in V$ such that $u<v$ set

$$
\Delta_{u v} \equiv 2 \frac{|\omega(\varphi(u) \varphi(v))|}{\|u\|\|v\|}=2 \frac{\left|\Omega_{u v}\right|}{\|u\|\|v\|}
$$

and for any graph $p$ on $V$ set

$$
\Delta(p) \equiv \prod_{\substack{u v \in p \\ u<v}} \Delta_{u v} .
$$

Note that $\Delta_{u v}$ and hence $\Delta(p)$ take values in the interval $[0,1]$. The following lemma, which controls the contribution of the exit graph $g$ to the sum (4.59) is immediate.

Lemma 4.6 For any $p \in \mathcal{P}(W)$

$$
|\Omega(p)| \leq 2^{-|W| / 2} \Delta(p)\left(\prod_{w \in W}\|w\|\right) .
$$

Applying this bound to $g \in \operatorname{Ex}(\Pi)$ and using Lemma 4.4 we finally get from Formula (4.59):

Lemma 4.7 Under the hypotheses of Lemma 4.2 the following estimate holds

$$
\left|\omega_{T}\left(A_{1}, \ldots, A_{n}\right)\right| \leq 2^{-|V| / 2}\left(\prod_{v \in V}\|v\|\right) \sum_{g \in \operatorname{Ex}(\Pi)}|\Theta(g)| \Delta(g) .
$$

\subsection{Counting exit graphs and their admissible partitions}

Lemma 4.8 For any ordered partition $\Pi$ of $V$ one has

$$
|\operatorname{Ex}(\Pi)| \leq 4^{|V|}|\Pi| !
$$

and for any $g \in \operatorname{Ex}(\Pi)$

$$
|\Theta(g)| \leq 4^{|V|}
$$

Proof. We set $|V|=2 N, \Pi=\left(V_{1}, \ldots, V_{n}\right)$ and $\left|V_{i}\right|=k_{i}$. To construct an exit graph we must first select $n$ exit points $x_{i} \in V_{i}$. Thus, there are $k_{1} k_{2} \cdots k_{n}$ exit points configurations. Each exit point $x_{i}$ has now to be paired with a different vertex $y_{i} \in V$, subject to some constraints. Releasing these constraints we obtain the upper bound $2 N(2 N-1) \cdots(2 N-n+1)$ on the number of such pairings. Thus,

$$
|\operatorname{Ex}(\Pi)| \leq 2 N(2 N-1) \cdots(2 N-n+1) k_{1} \cdots k_{n}=\left(\begin{array}{c}
2 N \\
n
\end{array}\right) k_{1} \cdots k_{n} n ! .
$$

The result follows from the facts that the binomial coefficient is bounded by $2^{2 N}$ and $k_{i} \leq 2^{k_{i}}$.

A $g$-admissible partition is a partition of $V(g)$ into four sets. Since there are $4^{|V(g)|}$ such partitions the second estimate follows. $\square$ 


\subsection{Proof of Theorem 3.5}

To prove Theorem 3.5 we set $A_{i}=\varphi\left(\mathrm{e}^{\mathrm{i} t_{i} h} f_{i 1}\right) \cdots \varphi\left(\mathrm{e}^{\mathrm{i} t_{i} h} f_{i k_{i}}\right)$ and apply Lemma 4.7 to the case

$$
V_{i} \equiv\left\{\mathrm{e}^{\mathrm{i} t_{i} h} f_{i 1}, \ldots, \mathrm{e}^{\mathrm{i} t_{i} h} f_{i k_{i}}\right\}, \quad i \in I \equiv\{1, \ldots, n\} .
$$

We set $2 N=|V|=\sum_{i} k_{i}$ and obtain

$$
\int_{[0, t]^{n}}\left|\omega_{T}\left(A_{1}, \ldots, A_{n}\right)\right| \mathrm{d} t_{1} \cdots \mathrm{d} t_{n} \leq 2^{-N}\left(\prod_{v \in V}\|v\|\right) \sum_{g \in \operatorname{Ex}(\Pi)}|\Theta(g)| C(g),
$$

where

$$
C(g) \equiv \int_{[0, t]^{n}} \Delta(g) \mathrm{d} t_{1} \cdots \mathrm{d} t_{n}
$$

Lemma 4.9 Let $g$ be a graph with vertex set $V$. Denote by $N_{c}(g)$ the number of connected components of its skeleton $g / \Pi$. Then one has

$$
\int_{[0, t]^{n}} \Delta(g) \mathrm{d} t_{1} \cdots \mathrm{d} t_{n} \leq C^{n-N_{c}(g)} t^{N_{c}(g)},
$$

with

$$
C \equiv \max \left(1, \max _{i j k l} \frac{2}{\left\|f_{i j}\right\|\left\|f_{k l}\right\|} \int_{-\infty}^{\infty}\left|\omega\left(\varphi\left(f_{i j}\right) \tau^{t}\left(\varphi\left(f_{k l}\right)\right)\right)\right| \mathrm{d} t\right) .
$$

Proof. Assume first that the skeleton $g / \Pi$ is connected. Then it has a spanning tree $(\Pi, T)$. Fix a root $V_{r}$ in $T$ and for $j \in I \backslash\{r\}$ let $V_{l(j)}$ be the parent of $V_{j}$ in $T$. Let $\pi \in S_{n}$ be a relabeling of the vertices of $T$ such that $\pi(r)=1$ and $\pi(l(j))<\pi(j)$ for $j \in I \backslash\{r\}$. Define new variables by $s_{j}=t_{j}-t_{l(j)}$ for $j \in I \backslash\{r\}$ and $s_{r}=t_{r}$. The corresponding Jacobian matrix is $J_{i j}=\delta_{i j}-\left(1-\delta_{i r}\right) \delta_{l(i) j}$. By our choice of the relabeling $\pi$ the reordered matrix

$$
J_{i j}^{\prime}=J_{\pi^{-1}(i) \pi^{-1}(j)}=\delta_{i j}-\left(1-\delta_{i 1}\right) \delta_{\pi\left(l\left(\pi^{-1}(i)\right)\right) j},
$$

is lower triangular with ones on the diagonal. Thus the Jacobian determinant is given by $|\operatorname{det} J|=\left|\operatorname{det} J^{\prime}\right|=1$.

For each edge $V_{j} V_{l(j)} \in T$ there is a corresponding edge $e_{j}=u_{j} v_{j} \in g$ with $u_{j}=\mathrm{e}^{\mathrm{i} t_{j} h} f_{j, a_{j}} \in V_{j}$ and $v_{j}=\mathrm{e}^{\mathrm{i} t_{l(j)} h} f_{l(j) b_{j}} \in$ $V_{l(j)}$ and therefore a factor

$$
\Delta_{e_{j}}\left(s_{j}\right)=\frac{2}{\left\|f_{j a_{j}}\right\|\left\|f_{l(j) b_{j}}\right\|}\left\{\begin{array}{ll}
\left|\omega\left(\varphi\left(f_{j a_{j}}\right) \tau^{-s_{j}}\left(\varphi\left(f_{l(j) b_{j}}\right)\right)\right)\right| & \text { for } j<l(j), \\
\left|\omega\left(\varphi\left(f_{l(j) b_{j}}\right) \tau^{s_{j}}\left(\varphi\left(f_{j a_{j}}\right)\right)\right)\right| & \text { for } j>l(j),
\end{array},\right.
$$

in $\Delta(g)$. It follows that

$$
\Delta(g) \leq \prod_{j \in I \backslash\{r\}} \Delta_{e_{j}}\left(s_{j}\right)
$$

and hence

$$
\int_{[0, t]^{n}} \Delta(g) \mathrm{d} t_{1} \cdots \mathrm{d} t_{n} \leq \int_{0}^{t}\left(\prod_{j \in I \backslash\{r\}} \int_{-t}^{t} \Delta_{e_{j}}\left(s_{j}\right) \mathrm{d} s_{j}\right) \mathrm{d} s_{r} \leq C^{n-1} t .
$$

In the general case, $g / \Pi$ is the disjoint union of $N_{c}(g)$ connected subgraphs. Applying the above estimate to each of them yields the result. $\square$

Inserting the estimate (4.65) into Equ. (4.64) and using Lemma 4.8 we finally obtain, taking into account the fact that the skeleton of an exit graph can have at most $n / 2$ connected components

$$
\int_{[0, t]^{n}}\left|\omega_{T}\left(A_{1}, \ldots, A_{n}\right)\right| \mathrm{d} t_{1} \cdots \mathrm{d} t_{n} \leq\left(8 \sqrt{2} \max _{i j}\left\|f_{i j}\right\|\right)^{2 N} C^{n} t^{n / 2} n !,
$$

which concludes the proof of Part 1 . 


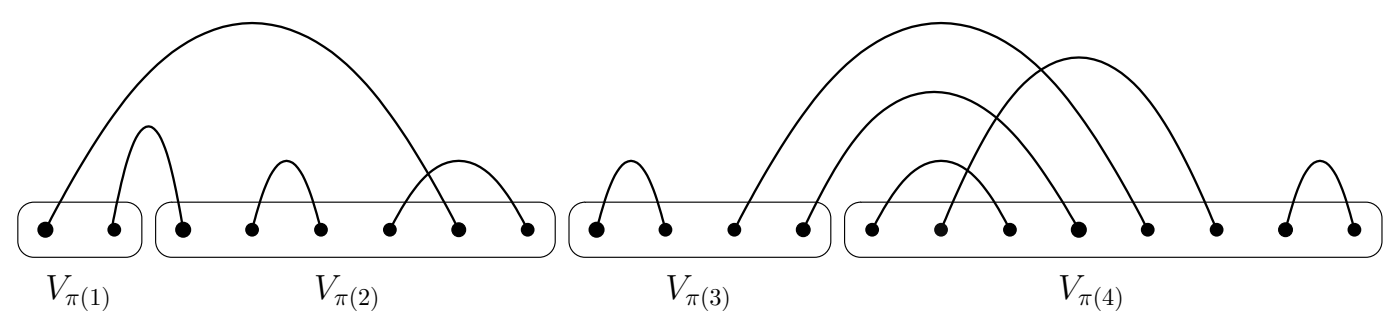

Figure 5: The pairing $\pi$ induced by a maximally disconnected pairing $p$.

To prove part 2 it suffices to notice that if $n$ is odd then the skeleton of an exit graph can have at most $(n-1) / 2$ connected components.

To prove part 3, we go back to Formula (4.57) and write

$$
t^{-n / 2} \int_{[0, t]^{n}} \omega_{T}\left(A_{1}, \ldots, A_{n}\right) \mathrm{d} t_{1} \cdots \mathrm{d} t_{n}=\sum_{p \in \mathcal{P}(\Pi)} \varepsilon(p) t^{-n / 2} \int_{[0, t]^{n}} \Omega(p) \mathrm{d} t_{1} \cdots \mathrm{d} t_{n} .
$$

By Lemmata 4.6 and 4.9 one has, as $t \rightarrow \infty$,

$$
t^{-n / 2} \int_{[0, t]^{n}} \Omega(p) \mathrm{d} t_{1} \cdots \mathrm{d} t_{n}=O\left(t^{N_{c}(p)-n / 2}\right) .
$$

Thus, the pairings $p \in \mathcal{P}(\Pi)$ which contribute to the limit $t \rightarrow \infty$ are maximally disconnected in the sense that their skeleton have exactly $n / 2$ connected components. The skeleton $p / \Pi$ of such a pairing induces a pairing $\pi \in \mathcal{P}_{n / 2}$ such that

$$
p=p_{1} \vee \cdots \vee p_{n / 2}, \quad p_{j} \in \mathcal{P}_{0}\left(V_{\pi(2 j-1)}, V_{\pi(2 j)}\right),
$$

where $\mathcal{P}_{0}\left(V_{i}, V_{j}\right)$ denotes the set of pairings on $V_{i} \cup V_{j}$ whose skeleton w.r.t. the partition $\left(V_{i}, V_{j}\right)$ has no isolated vertex (see Figure 5). Since the map $p \mapsto\left(\pi, p_{1}, \ldots, p_{n / 2}\right)$ is clearly bijective we can, for the purpose of computing the limit of (4.66) as $t \rightarrow \infty$, replace $\omega_{T}\left(A_{1}, \ldots, A_{n}\right)$ by

$$
\sum_{\pi \in \mathcal{P}_{n / 2}} \sum_{p_{j} \in \mathcal{P}_{0}\left(V_{\pi(2 j-1)}, V_{\pi(2 j)}\right)} \varepsilon\left(p_{1} \vee \cdots \vee p_{n / 2}\right) \Omega\left(p_{1} \vee \cdots \vee p_{n / 2}\right) .
$$

By Lemma 4.1 we have

$$
\varepsilon\left(p_{1} \vee \cdots \vee p_{n / 2}\right)=\varepsilon\left(V_{\pi(1)}, \ldots, V_{\pi(n)}\right) \varepsilon\left(p_{1}\right) \cdots \varepsilon\left(p_{n / 2}\right), \quad \Omega\left(p_{1} \vee \cdots \vee p_{n / 2}\right)=\Omega\left(p_{1}\right) \cdots \Omega\left(p_{n / 2}\right)
$$

and by the remark following it $\varepsilon\left(V_{\pi(1)}, \ldots, V_{\pi(n)}\right)=1$. Thus, the last expression can be rewritten as

$$
\sum_{\pi \in \mathcal{P}_{n / 2}} \prod_{j=1}^{n / 2}\left(\sum_{p_{j} \in \mathcal{P}_{0}\left(V_{\pi(2 j-1)}, V_{\pi(2 j)}\right)} \varepsilon\left(p_{j}\right) \Omega\left(p_{j}\right)\right) .
$$

Finally observe that, by Lemma 4.2,

$$
\sum_{p \in \mathcal{P}_{0}\left(V_{i}, V_{j}\right)} \varepsilon(p) \Omega(p)=\omega_{T}\left(A_{i}, A_{j}\right) .
$$

One easily concludes the proof by the remark following Theorem 3.5 and the dominated convergence theorem.

\section{References}

[AJPP1] Aschbacher, W., Jakšić, V., Pautrat, Y., Pillet, C.-A.: Topics in non-equilibrium quantum statistical mechanics. In Open Quantum Systems III. S. Attal, A. Joye, C.-A. Pillet editors. Lecture Notes in Mathematics 1882, Springer, New York (2006). 
[AJPP2] Aschbacher, W., Jakšić, V., Pautrat, Y., Pillet, C.-A.: Transport properties of quasi-free fermions. J. Math. Phys. 48, 032101 (2007).

[AJPP3] Aschbacher, W., Jakšić, V., Pautrat, Y., Pillet, C.-A.: Fluctuations in quasi-free fermionic systems. In preparation.

[AM] Aizenstadt, V.V., Malyshev, V.A.: Spin interaction with an ideal Fermi gas. J. Stat. Phys. 48, 51 (1987).

[Bil] Billingsley, P.: Probability and Measure Wiley, New York (1979).

[BGM] Botvich, D.D., Guta, M., Maassen, H.: Stability of Bose dynamical systems and branching theory. Preprint (mp_arc 99-130).

[BM1] Botvich, D.D., Malyshev, V.A.: Unitary equivalence of temperature dynamics for ideal and locally perturbed Fermi gas. Commun. Math. Phys. 61, 209 (1983).

[BM2] Botvich, D.D., Malyshev, V.A.: Asymptotic completeness and all that for an infinite number of fermions. In ManyParticle Hamiltonians: Spectra and Scattering. Minlos, R. A. editor. Advances in Soviet Mathematics 5, 39, AMS, Providence (1991).

[BKR] Bratteli, O., Kishimoto, A., Robinson, D.W.: Stability properties and the KMS condition. Commun. Math. Phys. 61, 209 (1978).

[BR1] Bratteli, O., Robinson, D. W.: Operator Algebras and Quantum Statistical Mechanics 1. Springer, Berlin (1987).

[BR2] Bratteli, O., Robinson, D. W.: Operator Algebras and Quantum Statistical Mechanics 2. Second edition, Springer, Berlin (1996).

[CH] Cushen, C.D., Hudson, R. L.: A quantum-mechanical central limit theorem, J. Appl. Prob. 8, 454 (1971).

[Da1] Davies, E.B.: Quantum Theory of Open Systems. Academic Press, London (1976).

[Da2] Davies, E.B.: Markovian master equations. Commun. Math. Phys. 39, 91 (1974).

[DGM] De Groot, S.R., Mazur, P.: Non-Equilibrium Thermodynamics. North-Holland, Amsterdam (1969).

[De1] Dereziński, J.: Boson free fields as a limit of fields of a more general type. Rep. Math. Phys. 21, 405 (1985).

[De2] Dereziński, J.: Introduction to representations of canonical commutation and anticommutation relations. In Large Coulomb Systems-Quantum Electrodynamics. J. Dereziński and H. Siedentop editors. Lecture Notes in Physics 695, Springer, New York (2006).

[E] Ellis, R.S.: Entropy, Large Deviations, and Statistical Mechanics. Springer, New-York (1985).

[FMU] Fröhlich, J., Merkli, M., Ueltschi, D.: Dissipative transport: thermal contacts and tunneling junctions. Ann. Henri Poincaré 4, 897 (2004).

[GV] Goderis, D., Vets, P.: Central limit theorem for mixing quantum systems and the CCR algebra of fluctuations. Commun. Math. Phys. 122, 249 (1989).

[GVV1] Goderis, D., Verbeure, A., Vets, P.: Noncommutative central limits. Probab. Theory Related Fields 82, 527 (1989).

[GVV2] Goderis, D., Verbeure, A., Vets, P.: Quantum central limit and coarse graining. In Quantum probability and applications, $V$. Lecture Notes in Math., 1442, 178 (1988).

[GVV3] Goderis, D., Verbeure, A., Vets, P.: About the mathematical theory of quantum fluctuations. In Mathematical Methods in Statistical Mechanics. Leuven Notes Math. Theoret. Phys. Ser. A Math. Phys., 1, 31. Leuven Univ. Press, Leuven (1989).

[GVV4] Goderis, D., Verbeure, A., Vets, P.: About the exactness of the linear response theory. Commun. Math. Phys. 136, 265 (1991).

[GVV5] Goderis, D., Verbeure, A., Vets, P.: Theory of quantum fluctuations and the Onsager relations. J. Stat. Phys. 56, 721 (1989).

[GVV6] Goderis, D., Verbeure, A., Vets, P.: Dynamics of fluctuations for quantum lattice systems. Commun. Math. Phys. 128, 533 (1990).

[GvW] Giri, N., von Waldenfels, W., : An algebraic version of the central limit theorem. Z. für Warscheinlichkeitstheorie 42, 129 (1978).

[Ha1] Haag, R.: Quantum field theories with composite particles and asymptotic completeness. Phys. Rev. 112, 669 (1958). 
[Ha2] Haag, R.: The framework of quantum field theory. Nuovo Cimento Supp. 14, 131 (1959).

[HL1] Hepp, K., Lieb, E.H.: Equilibrium statistical mechanics of matter interacting with the quantized radiation field. Phys. Rev. A8, 2517 (1973).

[HL2] Hepp, K., Lieb, E.H.: Phase transitions in reservoir driven open systems with applications to lasers and superconductivity. Helv. Phys. Acta 46, 573 (1973).

[JKP] Jakšić, V., Kritchevski, E., Pillet, C.-A.: Mathematical theory of the Wigner-Weisskopf atom. In Large Coulomb Systems-Quantum Electrodynamics. J. Dereziński and H. Siedentop editors. Lecture Notes in Physics 695, Springer, New York (2006).

[JOP1] Jakšić, V., Ogata, Y., Pillet, C.-A.: The Green-Kubo formula and the Onsager reciprocity relations in quantum statistical mechanics. Commun. Math. Phys. 265, 721 (2006).

[JOP2] Jakšić, V., Ogata, Y., Pillet, C.-A.: Linear response theory for thermally driven quantum open systems. J. Stat. Phys. 123, 547, (2006).

[JOP3] Jakšić, V., Ogata, Y., Pillet, C.-A.: The Green-Kubo formula for the spin-fermion system. Commun. Math. Phys. 268, 401 (2006).

[JOP4] Jakšić, V., Ogata, Y., Pillet, C.-A.: The Green-Kubo formula for locally interacting fermionic open systems. Ann. Henri Poincaré 8, 1013 (2007).

[JPP] Jakšić, V., Pautrat, Y., Pillet, C.-A.: A non-commutative Lévy-Cramér theorem. Preprint.

[JP1] Jakšić, V., Pillet, C-A.: On entropy production in quantum statistical mechanics. Commun. Math. Phys. 217, 285 (2001).

[JP2] Jakšić, V., Pillet, C.-A.: Non-equilibrium steady states for finite quantum systems coupled to thermal reservoirs. Commun. Math. Phys. 226, 131 (2002).

[JP3] Jakšić, V., Pillet, C.-A.: Mathematical theory of non-equilibrium quantum statistical mechanics. J. Stat. Phys. 108, 787 (2002).

[JP4] Jakšić, V., Pillet, C-A.: A note on the entropy production formula. Contemp. Math. 327, 175 (2003).

[JP5] Jakšić, V., Pillet, C.-A.: On the strict positivity of entropy production. To appear in Contemp. Math. (2007).

[JP6] Jakšić, V., Pillet, C.-A.: In preparation.

[KL] Kipnis, C., Landim, C.: Scaling Limits of Interacting Particle Systems. Springer, Berlin (1999).

[KTH] Kubo, R., Toda, M., Hashitsune, N.: Statistical Physics II. Second edition, Springer, Berlin (1991).

[Kup] Kuperberg, G., .

[Li] Liverani, C.: Central limit theorem for deterministic systems. International Conference on Dynamical Systems (Montevideo, 1995), 56-75, Pitman Res. Notes Math. Ser., 362, Longman, Harlow, (1996).

[Ma1] Matsui, T.: Bosonic central limit theorem for the one-dimensional XY model. Rev. Math. Phys. 14, 675 (2002).

[Ma2] Matsui, T.: On the algebra of fluctuation in quantum spin chains. Ann. Henri PoincarÃ@) 4, 63 (2003).

[Me] Meyer, Y.: Quantum Probability for Probabilists. Lecture Notes in Mathematics 1358. Springer Verlag, Berlin, 1993.

[MSTV] Manuceau, J., Sirugue, M., Testard, D., Verbeure, A.: The smallest $C^{*}$-algebra for canonical commutations relations. Commun. Math. Phys. 32, 271 (1973).

[OP] Ohya, M., Petz, D.: Quantum Entropy and its Use. Springer, Berlin (1993).

[Pe] Petz, D.: An invitation to the algebra of canonical commutation relations. Leuven Notes in Mathematical and Theoretical Physics. Series A: Mathematical Physics, 2. Leuven University Press, Leuven (1990).

[Pi] Pillet, C-A.: Quantum dynamical systems. In Open Quantum Systems I. S. Attal, A. Joye, C.-A. Pillet editors. Lecture Notes in Mathematics 1880, Springer, New York (2006).

[Ro] Robinson, D.W.: Return to equilibrium. Commun. Math. Phys. 31, 171 (1973).

[Ru1] Ruelle, D: On the asymptotic condition in quantum field theory. Helv. Phys. Acta 35, 147 (1962).

[Ru2] Ruelle, D.: Natural nonequilibrium states in quantum statistical mechanics. J. Stat. Phys. 98,57 (2000).

[Ru3] Ruelle, D.: Entropy production in quantum spin systems. Commun. Math. Phys. 224, 3 (2001).

[Ru4] Ruelle, D.: Topics in quantum statistical mechanics and operator algebras. Preprint, mp-arc 01-257 (2001).

[Sp] Spohn, H.: Large Scale Dynamics of Interacting Particles. Texts and Monographs in Physics, Springer, Berlin (1991). 